\title{
29. FORAMINIFER PRESERVATION RECORD FOR THE LAST MILLION YEARS: SITE 805, ONTONG JAVA PLATEAU ${ }^{1}$
}

\author{
M. Yasuda, ${ }^{2}$ W.H. Berger, ${ }^{2}$ G. Wu, ${ }^{2,4}$ S. Burke, ${ }^{2}$ and H. Schmidt ${ }^{3}$
}

\begin{abstract}
Downcore changes in various carbonate dissolution indexes are documented for Hole $805 \mathrm{C}$ for the last $1.2 \mathrm{~m} . \mathrm{y}$. These indexes include degree of fragmentation of planktonic foraminifers, percent sand, abundance ratio of species of contrasting solution susceptibilities (Globigerinoides sacculifer vs. Pulleniatina, Globorotalia tumida, and Globorotalia menardii), and the difference in $\delta^{18} \mathrm{O}$ between species of contrasting solution susceptibilities ( $G$. sacculifer vs. Pulleniatina). These preservation indexes have been combined into a single composite dissolution index that corresponds closely to the $\delta^{18} \mathrm{O}$ record. The rate of change of the oxygen isotope signal is also important, with glacial-to-interglacial transitions corresponding to maximum preservation events and vice versa. For information on changing productivity (which is important because an increased supply of organic matter may enhance dissolution by lowering $\mathrm{pH}$ upon degradation), we present the abundance of coarse-fraction benthic foraminifers per gram and the ratio between two planktonic foraminiferal species, one of which is strongly associated with equatorial upwelling (Globorotalia tumida vs. Pulleniatina). Our results suggest that productivity plays a subordinate role in determining foraminifer preservation. Furthermore, our results confirm previous observations that associate enhanced preservation events with glacial periods and with glacial-to-interglacial transitions. A correlation between preservation and sedimentation rates of these carbonate-rich sediments could not be established. Notable differences are present between the responses of individual dissolution indexes, indicating that processes other than dissolution determine proxy indexes to varying degrees.
\end{abstract}

\section{INTRODUCTION}

The carbonate cycles in Quaternary deep-sea sediments of the equatorial Pacific were discovered some 40 yr ago during the Swedish Deep-Sea Expedition and stimulated thinking about cyclic changes in ocean productivity driven by glacial-interglacial changes in the intensity of upwelling (Arrhenius, 1952). The exact mode of origin of these carbonate cycles is still unknown even though much study since their discovery has produced detailed information on carbonate distributions and on stable isotope and preservation stratigraphies in both the eastern and western equatorial Pacific (Hays et al., 1969; Berger, 1973; Shackleton and Opdyke, 1973, 1976; Thompson and Saito, 1974; Luz and Shackleton, 1975; Pisias, 1976; Thompson, 1976; Moore et al., 1977, 1982; Valencia, 1977; Adelseck, 1977; Adelseck and Anderson, 1978; Volat et al., 1980; Gardner, 1982; Schiffelbein, 1984; Schiffelbein and Dorman, 1986; Crowley, 1985; Chuey et al., 1987; Lyle et al., 1988; Farrell and Prell, 1989, 1991; Wu and Berger, 1989, 1991; Hebbeln et al., 1990; Wu et al., 1990, 1991; Grötsch et al., 1991; Le and Shackleton, 1992).

Discussions have centered on quantifying the relative importance of fluctuations in supply vs. dissolution in determining percent carbonate because of interest in the marine carbon cycle. Much evidence exists that basin-wide dissolution cycles have produced correlatable carbonate cycles within a glacial/interglacial time frame (Berger and Vincent, 1981; Vincent, 1985). Equally strong evidence exists in favor of the productivity concept (Arrhenius, 1952, 1988). The question cannot be answered from a knowledge of carbonate cycles alone, if the limits allowed for productivity variations are suitably wide (Berger, 1992). Recent calculations by Archer (1991) suggest that a twofold increase in glacial production is sufficient to explain the

\footnotetext{
${ }^{1}$ Berger, W.H., Kroenke, L.W., Mayer, L.A., et al., 1993. Proc. ODP, Sci. Results, 130: College Station, TX (Ocean Drilling Program).

${ }^{2}$ Geological Research Division, Scripps Institution of Oceanography, University of California, San Diego, La Jolla, CA 92093, U.S.A.

${ }^{3}$ Fachbereich Geowissenschaften, Universität Bremen, Postfach 330440, 2800 Bremen 33, Federal Republic of Germany.

${ }^{4}$ Present address: Université du Québec à Montréal, GEOTOP, Case postale 8888 , succursale A, Montréal, Québec, H3C 3P8, Canada.
}

variations in carbonate accumulation rates, although concurrent changes in Pacific deep-water carbonate saturation are not discounted by him. Archer's estimate of the range of glacial/interglacial productivity is within the range of variation found in the modern ocean, unlike some previous estimates. Herguera and Berger (1991) suggest factors of 1.6-2.0 for the glacial vs. postglacial productivity contrast on Ontong Java Plateau. To complicate matters, net carbonate accumulation is also affected by increased organic-matter supply, which is presumably positively correlated with increased carbonate supply. Oxidation of organic matter $\left(\mathrm{C}_{\mathrm{org}}\right)$ should increase carbonate dissolution by lowering $\mathrm{pH}$ at and immediately below the seafloor. However, this dissolution mechanism may only be important if $\mathrm{C}_{\mathrm{org}}$ is not oxidized before being incorporated into the mixed layer (Archer, 1991). Thus, this process may be of decreasing importance as depth to seafloor increases. Redeposition processes must also be considered. Differences in carbonate content between Leg 130 sites at different depths are insufficient to explain differences in sedimentation rates, if carbonate dissolution is assumed to be the cause of the depth gradient in sediment accumulation (Berger et al., 1991). Thus, the fate of noncarbonate dilutants or winnowing must be important and changes in the intensity of these factors must play a role in producing the cycles.

In the western equatorial Pacific, at depths above the lysocline, carbonate content is uniformly high (near $85 \%-90 \%$ ) in the Quaternary, and fluctuations are low (typically, within a range of about $6 \%$ ). Changes in the percentages of $\mathrm{CaCO}_{3}$ are not a sensitive indicator of the history of carbonate dissolution at these high carbonate concentrations, even when complications introduced by fluctuations in dilution with noncarbonate material can be assumed to be at a minimum, as at this site. Therefore, we construct a stratigraphy of foraminifer preservation in the western equatorial Pacific. If the preservational signal may be interpreted in terms of saturation of deep waters, at least in large part (Berger, 1977; Berger and Keir, 1984), in principle it should be possible to reconstruct the fluctuations of deep-water saturation (Keir and Berger, 1983; Peterson and Prell, 1985a, 1985b; Hebbeln et al., 1990; Grötsch et al., 1991). We also construct a stratigraphy of productivity, based on the abundance of benthic foraminifers and changes in the composition of the pelagic assemblage, in order to estimate the importance of productivity to carbonate preservation. 
We report here on the upper $20 \mathrm{~m}$ recovered from Hole $805 \mathrm{C}$, comprising Cores $130-805 \mathrm{C}-1 \mathrm{H}$ and $-2 \mathrm{H}$, and the upper portion of Core $130-805 \mathrm{C}-3 \mathrm{H}$. Site 805 was drilled during Ocean Drilling Program (ODP) Leg 130 , at $1^{\circ} 13.9^{\prime} \mathrm{N}, 160^{\circ} 31.77^{\prime} \mathrm{E}$, in $3188 \mathrm{~m}$ of water. This depth is just above the present regional lysocline (near $3.3 \mathrm{~km}$; Wu and Berger, 1991). We also use data from Hole 806B for comparison (from Berger et al., this volume). Site 806 was drilled at $00^{\circ} 10.11^{\prime} \mathrm{N}, 159^{\circ} 21.69^{\prime} \mathrm{E}$, in $2520 \mathrm{~m}$ of water. The location of the two sites is shown in Figure 1.

\section{MEASUREMENTS}

Samples used in this study are from Hole $805 \mathrm{C}$, from the sediment surface down through the upper portion of Core 130-805C-3H near $20 \mathrm{~m}$, except for measurements involving planktonic foraminifer counts, which are from the sediment surface down through the bottom of Core $130-805 \mathrm{C}-2 \mathrm{H}$ near $17 \mathrm{~m}$. The typical sample spacing is $10 \mathrm{~cm}$. The Brunhes/Matuyama boundary was detected at $12.7 \mathrm{~m}$ (official ODP depth) within Core 130-805C-2H (Shipboard Scientific Party, 1991). Assuming an age of $790,000 \mathrm{yr}$ for the Brunhes/Matuyama boundary (Shackleton et al., 1990; Izett and Obradovich, 1991), the average sedimentation rate above this boundary is near $1.6 \mathrm{~cm} / \mathrm{k} . \mathrm{y}$. and the typical sampling interval represents $6000 \mathrm{yr}$. The age of the oldest sediment studied, at $20 \mathrm{~m}$ below seafloor (mbsf), is estimated to be near 1.2 m.y., based on linear extrapolation of the sedimentation rate above the Brunhes/Matuyama boundary.

Our measurements of dissolution characteristics include degree of planktonic foraminifer fragmentation; relative abundance of whole Globigerinoides sacculifer vs. the sum of whole Globigerinoides sacculifer, whole Pulleniatina, whole Globorotalia tumida, and whole Globorotalia menardii; and percent sand-size fragments (Table 1). In addition, the $\delta^{18} \mathrm{O}$ record of planktonic foraminifers (Berger et al., this volume) is used to interpret the dissolution stratigraphy. Although benthic foraminifer abundance has been correlated with intensity of dissolution (Parker and Berger, 1971; Thunell, 1976), it is not used here as a preservation index because of the strong productivity signal carried by this variable (Herguera and Berger, 1991). As mentioned, percent carbonate is not a very sensitive measure of dissolution above the lysocline on Ontong Java Plateau; therefore, we do not use it as a dissolution index. Within our sampling interval, the percent carbonate fluctuates in a narrow range from $84 \%$ to $92 \%$ (Shipboard Scientific Party, 1991).

To check whether any substantial relationship exists between productivity and dissolution, we use two productivity proxies: the total abundance of benthic foraminifers and the relative abundance of whole Globorotalia tumida vs. the sum of whole Pulleniatina and whole $G$. tumida. These productivity indexes are nevertheless susceptible to dissolution effects. However, all species used in these productivity indexes are quite resistant to dissolution (Parker and Berger, 1971). Little preserved organic matter is present at our site. $C_{\text {org }}$ values measured within the top meter of sediments at Ontong Java Plateau generally range between $0.1 \%$ and $0.3 \%$ (Price, 1988).

\section{SAMPLE PREPARATION}

For each sample, approximately $10 \mathrm{~cm}^{3}$ of wet-bulk sediment were freeze dried and then weighed. All samples were disaggregated by soaking in a mixture of buffered CALGON solution and $5 \%$ hydrogen peroxide for approximately $1 \mathrm{hr}$. Samples were then wet sieved through a $63-\mu \mathrm{m}$ mesh sieve for approximately $20 \mathrm{~min}$, with an intervening 5-s ultrasonification to loosen small particles trapped inside individual tests. Samples were given a final 1-min rinse in deionized water. Samples were then dried overnight (or longer) at $55^{\circ} \mathrm{C}$ and then weighed to determine the weight percent of particles greater than sand size $(>63 \mu \mathrm{m})$. Abundance of total benthic foraminifers was determined using the size fraction $>250 \mu \mathrm{m}$. The fraction of planktonic foraminifer fragments and whole tests was determined in the size class $250-425 \mu \mathrm{m}$. The ratios of $G$, sacculifer vs. the sum of $G$. sacculifer, Pulleniatina, G. menardii, and $G$. tumida; and $G$. tumida vs. Pulleniatina plus $G$. tumida were determined using the size fraction $355-425 \mu \mathrm{m}$.

\section{RESULTS}

\section{Preservation Indexes}

Our measurements of indexes of preservation and productivity for Hole 805C are listed in Tables 1 and 2 according to official ODPdepths. All raw data are available from the first author. Preservation indexes are plotted vs. corrected depth in Figure 2. Our preservation indexes in Figure 2 look quite similar at a glance, except for the difference in $\delta^{18} \mathrm{O}$ between $G$. sacculifer and Pulleniatina (marked "POX-SOX" on the graph, data from Berger et al., this volume). The oxygen isotope record of Pulleniatina (POX) is given for guidance. The record of this species is somewhat less sensitive to the effects of differential dissolution than that of G. sacculifer (Wu and Berger, 1989).

A depth correction has been applied to all data displayed in the following graphs to avoid the apparent overlap of cores that occurs when using official ODP depth assignments. Depth corrections are necessitated because of the recovery of excess core (presumably from core expansion) and because of sampling gaps. Sampling gaps have been identified by comparison of matching physical properties between offset cores. Gaps between cores were estimated as $30 \mathrm{~cm}$ (Berger et al., this volume), based on comparisons of $\delta^{18} \mathrm{O}$ records for Hole $805 C$ with those of nearby Cores V28-238 and V28-239 and on visual comparisons of GRAPE data for offset Holes 805A, 805B, and $805 \mathrm{C}$. After adding back the presumed length of missing core, excess core recovery was adjusted to fit within official ODP core length assignments based on the assumption that the official depths at the tops of all cores is correct. In summary, the official ODP core depths were corrected by applying the following factors: $\mathrm{HI}=0.9615$ (ODP depth), $\mathrm{H} 2=0.9312$ (ODP depth), and $\mathrm{H} 3=0.9787$ (ODP depth). A more detailed description of the Hole $805 \mathrm{C}$ depth correction is given in Berger et al. (this volume).

For discussion in terms of age, we include an estimation of age vs. depth based on counting obliquity and eccentricity cycles. Our age assignments are listed in Table 3 . The 100- and 41-k.y. wavelength components of the $\delta^{18} \mathrm{O}$ Pulleniatina record were reconstructed by extracting the signal within $a \pm 15 \%$ band of the expected harmonics containing these components. The reconstructed 100 - and 41-k.y. signals are shown in Figure 3. By counting crests in the obliquity-related signal, we were able to assign depth increments to time increments of 41-k.y. Assignments of 41-k.y. spacing were based on best agreement with the spacing of the 100-k.y. cycles. The age of the isotopic stage $16 / 15$ termination is near $623 \mathrm{k} . \mathrm{y}$. in our scale (Fig. 3), in good agreement with the assignment of 618 k.y. of Imbrie et al. (1984). Below this level, our age assignments for the same isotopic features are older than theirs. The method used here for extraction of the obliquity and eccentricity components is identical to that described in detail by Berger et al. (this volume), except that our data was processed as a single continuous data set rather than as three shorter data sets (individual cores). Our age model is intended as a rough guide only. We did not adopt existing age models because of the controversy surrounding the true age of the Brunhes/Matuyama boundary (see Shackleton et al., 1990; Izett and Obradovich, 1991).

\section{Fragmentation}

For the fragmentation record (Berger, 1970a; Thompson, 1976; Thunell, 1976), we use the percent abundance of whole tests of all planktonic foraminifers and the percent abundance of whole tests within the G. menardii complex (Globorotalia menardii and Globorotalia tumida) (Table 1 and Fig. 2). Fragmentation of the $G$. menardii complex closely parallels the general fragmentation record, 


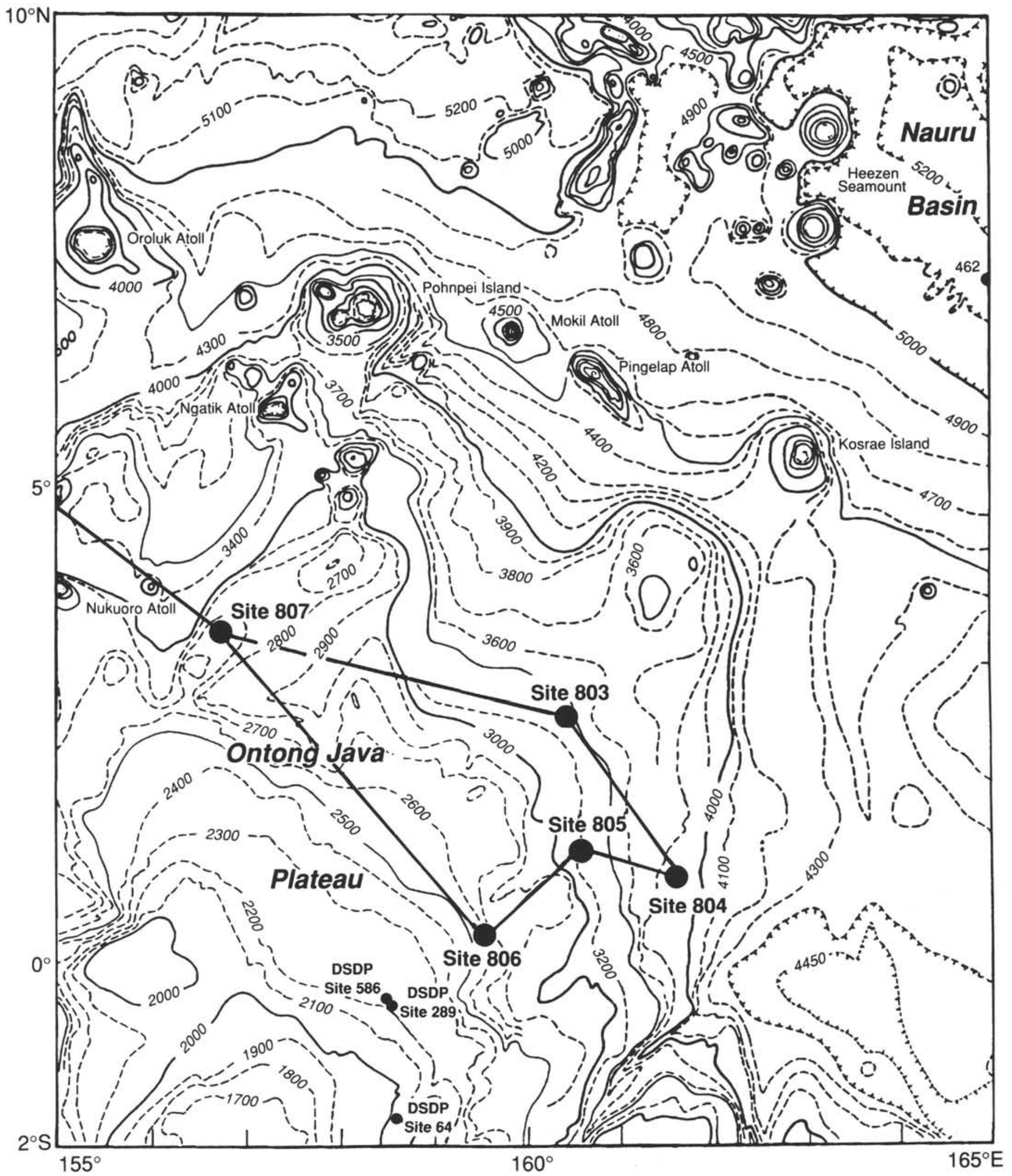

Figure 1. Location map. 
Table 1. Preservation indexes, Hole 805C.

\begin{tabular}{|c|c|c|c|c|c|c|c|c|c|c|c|}
\hline \multirow[b]{2}{*}{$\begin{array}{l}\text { Core, section, } \\
\text { interval }(\mathrm{cm})\end{array}$} & \multirow[b]{2}{*}{$\begin{array}{l}\text { Depth* } \\
\text { (mbsf) }\end{array}$} & \multirow[b]{2}{*}{$\begin{array}{c}\% \text { Sand } \\
\text { fraction } \\
>63 \mu \mathrm{m} \\
(\%)\end{array}$} & \multicolumn{2}{|c|}{ Fragmentation } & \multirow{2}{*}{$\begin{array}{c}\text { Planktonic } \\
\text { Ratio } \\
\text { [\#SACl } \\
\text { (\#SAC+ } \\
\text { \#PUL+ } \\
\text { \#MENA+ } \\
\text { \#TUMI)] }\end{array}$} & \multirow[b]{2}{*}{$\begin{array}{l}\text { Core, section, } \\
\text { interval }(\mathrm{cm})\end{array}$} & & & Fragmen & ation & $\begin{array}{c}\text { Planktonic } \\
\text { Ratio } \\
\text { [\#SACl }\end{array}$ \\
\hline & & & $\begin{array}{c}\text { Whole } \\
\text { G. menardii } \\
(\%)\end{array}$ & $\begin{array}{l}\text { Whole } \\
\text { tests } \\
(\%)\end{array}$ & & & $\begin{array}{l}\text { Depth* } \\
\text { (mbsf) }\end{array}$ & $\begin{array}{c}>63 \mu \mathrm{m} \\
(\%)\end{array}$ & $\begin{array}{l}\text { Whole } \\
\text { G. menardii } \\
(\%)\end{array}$ & $\begin{array}{l}\text { Whole } \\
\text { tests } \\
(\%)\end{array}$ & $\begin{array}{l}\text { \#PUL+ } \\
\text { \#MENA+ } \\
\text { \#TUMI)]† }\end{array}$ \\
\hline $1 \mathrm{H}-1,9-11$ & 0.09 & 25.18 & 20.2 & 42.9 & & $1 \mathrm{H}-5,49-51$ & 6.49 & 29.20 & 70.0 & 52.9 & 0.4 \\
\hline $1 \mathrm{H}-\mathrm{I}, 13-15$ & 0.13 & 44.72 & 42.6 & 71.9 & 0.3 & IH-5, 49-5I & 6.49 & & & & 0.4 \\
\hline $1 \mathrm{H}-\mathrm{I}, 19-21$ & 0.19 & 43.33 & 41.5 & 64.4 & & $1 \mathrm{H}-5,59-61$ & 6.59 & 23.26 & 58.1 & 51.5 & 0.2 \\
\hline IH-I, 19-21 & 0.19 & & 42.1 & 60.6 & & $1 \mathrm{H}-5,69-71$ & 6.69 & 21.17 & 65.8 & 57.3 & 0.2 \\
\hline $1 \mathrm{H}-1,21-23$ & 0.21 & 37.19 & 44.6 & 58.9 & 0.5 & IH-5, 79-81 & 6.79 & 21.40 & 61.4 & 52.4 & 0.2 \\
\hline $1 \mathrm{H}-1,29-31$ & 0.29 & 25.26 & 40.2 & 66.0 & 0.4 & 1H-5, 89-91 & 6.89 & 18.95 & 51.7 & 48.6 & 0.2 \\
\hline $1 \mathrm{H}-1,39-41$ & 0.39 & 29.76 & 53.6 & 50.8 & 0.3 & $1 \mathrm{H}-5,99-101$ & 6.99 & 15.95 & 40.1 & 51.7 & 0.2 \\
\hline IH-1, 39-41 & 0.39 & & & & & $1 \mathrm{H}-5,109-111$ & 7.09 & 19.32 & 28.1 & 39.8 & 0.2 \\
\hline $1 \mathrm{H}-1,49-51$ & 0.49 & 33.15 & 47.8 & 51.1 & 0.3 & $1 \mathrm{H}-5,119-121$ & 7.19 & 19.01 & 33.3 & 51.5 & 0.3 \\
\hline IH-1, 59-61 & 0.59 & 27.51 & 54.6 & 56.5 & 0.2 & $1 \mathrm{H}-5,119-121$ & 7.19 & & & & 0.3 \\
\hline IH-1, 69-71 & 0.69 & 24.95 & 34.0 & 47.4 & 0.2 & $\mid \mathrm{H}-5,129-131$ & 7.29 & 9.33 & 20.0 & 36.8 & 0.1 \\
\hline IH-1, 69-71 & 0.69 & & & & 0.2 & $1 \mathrm{H}-5,129-131$ & 7.29 & & 28.1 & 37.0 & \\
\hline IH-I, 75-77 & 0.75 & 27.55 & & & & IH-5, 142-145 & 7.42 & 9.58 & 14.9 & 17.1 & 0.1 \\
\hline 1H-1, 89-91 & 0.89 & 32.84 & 49.7 & 60.3 & 0.3 & $1 \mathrm{H}-5,149-151$ & 7.49 & 12.10 & 25.9 & 25.1 & 0.1 \\
\hline IH-1, 89-91 & 0.89 & & & & 0.3 & $2 \mathrm{H}-1,9-11$ & 7.95 & 18.63 & 47.0 & 52.3 & 0.1 \\
\hline 1H-1, 89-91 & 0.89 & & & & 0.3 & $2 \mathrm{H}-1,19-21$ & 7.99 & 16.41 & 39.9 & 52.3 & 0.2 \\
\hline IH-1,99-10I & 0.99 & 28.07 & 41.9 & 41.7 & 0.2 & $2 \mathrm{H}-1,29-31$ & 8.09 & 14.88 & 43.5 & 38.7 & 0.2 \\
\hline $1 \mathrm{H}-1,109-111$ & 1.09 & 19.52 & 35.9 & 32.9 & 0.1 & $2 \mathrm{H}-1,39-41$ & 8.19 & 7.88 & 29.8 & 41.6 & 0.1 \\
\hline $1 \mathrm{H}-1,119-121$ & 1.19 & 27.08 & 23.8 & 42.8 & 0.3 & $2 \mathrm{H}-1,49-51$ & 8.29 & 12.53 & 29.2 & 40.8 & 0.1 \\
\hline $1 \mathrm{H}-1,129-131$ & 1.29 & 40.77 & 44.2 & 58.9 & 0.5 & $2 \mathrm{H}-1,49-51$ & 8.29 & & & & 0.0 \\
\hline $1 \mathrm{H}-1,139-141$ & 1.39 & 19.91 & & & & $2 \mathrm{H}-1,59-61$ & 8.39 & 18.27 & 32.2 & 33.3 & 0.2 \\
\hline IH-I, 143-145 & 1.43 & 22.04 & 20.4 & 25.6 & 0.1 & $2 \mathrm{H}-1,69-71$ & 8.49 & 16.38 & 43.1 & 50.4 & 0.2 \\
\hline IH-2, 4-6 & 1.54 & 31.93 & 40.4 & 46.3 & 0.4 & $2 \mathrm{H}-1,79-81$ & 8.59 & 21.69 & 51.6 & 59.3 & 0.3 \\
\hline $1 \mathrm{H}-2,19-21$ & 1.69 & 18.63 & 22.9 & 20.9 & 0.1 & $2 \mathrm{H}-1,89-91$ & 8.69 & 11.61 & 41.9 & 48.9 & 0.1 \\
\hline $1 \mathrm{H}-2,29-31$ & 1.79 & 38.20 & 81.5 & 77.7 & 0.5 & $2 \mathrm{H}-1,99-101$ & 8.79 & 13.38 & 43.9 & 48.8 & 0.2 \\
\hline IH- $2,39-41$ & 1.89 & 40.73 & 63.0 & 69.0 & 0.4 & $2 \mathrm{H}-1,109-111$ & 8.89 & 11.89 & 43.5 & 45.6 & 0.1 \\
\hline $1 \mathrm{H}-2,49-51$ & 1.99 & 27.56 & 59.1 & 67.3 & 0.4 & $2 \mathrm{H}-1,119-121$ & 8.99 & 15.50 & 36.3 & 50.8 & 0.1 \\
\hline $1 \mathrm{H}-2,49-51$ & 1.99 & & & & 0.4 & $2 \mathrm{H}-1,129-131$ & 9.09 & 15.99 & 50.3 & 54.7 & 0.1 \\
\hline $\mathrm{IH}-2,59-61$ & 2.09 & 37.62 & 60.0 & 70.0 & 0.6 & $2 \mathrm{H}-1,139-141$ & 9.19 & 12.12 & 25.9 & 45.2 & 0.2 \\
\hline $1 \mathrm{H}-2,69-71$ & 2.19 & 37.88 & 54.5 & 62.4 & 0.6 & $2 \mathrm{H}-1,139-141$ & 9.19 & & & & 0.1 \\
\hline $1 \mathrm{H}-2,79-81$ & 2.29 & 25.80 & 53.2 & 60.3 & 0.5 & $2 \mathrm{H}-1,148-150$ & 9.28 & 20.34 & 48.6 & 51.4 & 0.3 \\
\hline $1 \mathrm{H}-2,89-91$ & 2.39 & 39.32 & 32.0 & 46.4 & 0.4 & $2 \mathrm{H}-2,9-11$ & 9.39 & 17.69 & 54.3 & 53.5 & 0.3 \\
\hline $1 \mathrm{H}-2,99-10 \mathrm{I}$ & 2.49 & 29.17 & 45.2 & 46.2 & 0.4 & $2 \mathrm{H}-2,19-21$ & 9.49 & 16.68 & 48.7 & 58.9 & 0.2 \\
\hline IH-2, 109-111 & 2.59 & 25.14 & 57.2 & 48.8 & 0.4 & $2 \mathrm{H}-2,29-31$ & 9.59 & 28.21 & 65.6 & 81.4 & 0.5 \\
\hline $1 \mathrm{H}-2,119-121$ & 2.69 & 15.65 & 30.6 & 47.6 & 0.3 & $2 \mathrm{H}-2,39-41$ & 9.69 & 31.09 & 73.4 & 77.7 & 0.4 \\
\hline $1 \mathrm{H}-2,129-131$ & 2.79 & 16.42 & 17.0 & 47.8 & 0.2 & $2 \mathrm{H}-2,49-51$ & 9.79 & 33.19 & 64.6 & 71.1 & 0.4 \\
\hline $\mathrm{IH}-2,143-145$ & 2.93 & 12.18 & & & & $2 \mathrm{H}-2,59-61$ & 9.89 & 29.91 & 76.8 & 76.2 & 0.3 \\
\hline $1 \mathrm{H}-2,148-150$ & 2.98 & 19.52 & 31.5 & 34.0 & 0.2 & $2 \mathrm{H}-2,69-71$ & 9.99 & 30.24 & 76.3 & 77.9 & 0.4 \\
\hline $1 \mathrm{H}-3,9-11$ & 3.09 & 37.47 & 41.4 & 54.4 & 0.7 & $2 \mathrm{H}-2,79-81$ & 10.09 & 28.69 & 65.9 & 72.5 & 0.4 \\
\hline $1 \mathrm{H}-3,19-21$ & 3.19 & 25.67 & 42.6 & 50.8 & 0.6 & $2 \mathrm{H}-2,89-91$ & 10.19 & 30.70 & 66.7 & 70.4 & 0.3 \\
\hline IH-3, 19-21 & 3.19 & & & & 0.7 & $2 \mathrm{H}-2,99-101$ & 10.29 & 28.83 & 69.6 & 74.4 & 0.3 \\
\hline $\mid \mathrm{H}-3,29-31$ & 3.29 & 17.38 & 49.8 & 52.2 & 0.5 & $2 \mathrm{H}-2,109-111$ & 10.39 & 33.71 & 63.3 & 76.8 & 0.3 \\
\hline IH-3, 39-41 & 3.39 & 30.42 & 52.2 & 42.5 & 0.5 & $2 \mathrm{H}-2,109-111$ & 10.39 & & 69.3 & 77.5 & \\
\hline $1 \mathrm{H}-3,49-51$ & 3.49 & 41.28 & 57.2 & 64.6 & 0.7 & $2 \mathrm{H}-2,119-12 \mathrm{I}$ & 10.49 & 28.44 & 80.4 & 78.1 & 0.3 \\
\hline $\mid \mathrm{H}-3,49-51$ & 3.49 & & 54.7 & 64.0 & & $2 \mathrm{H}-2,129-131$ & 10.59 & 30.12 & 61.5 & 75.7 & 0.3 \\
\hline $1 \mathrm{H}-3,59-61$ & 3.59 & 35.58 & & & & $2 \mathrm{H}-2,139-141$ & 10.69 & 25.85 & 53.5 & 71.1 & 0.3 \\
\hline $1 \mathrm{H}-3,69-71$ & 3.69 & 23.84 & 57.1 & 64.9 & 0.5 & $2 \mathrm{H}-2,148-150$ & 10.78 & 26.38 & 68.6 & 76.2 & 0.3 \\
\hline $\mid \mathrm{H}-3,79-81$ & 3.79 & 30.62 & 70.9 & 65.5 & 0.5 & $2 \mathrm{H}-3,9-11$ & 10.89 & 20.75 & 64.7 & 68.3 & 0.3 \\
\hline $1 \mathrm{H}-3,79-81$ & 3.79 & & 61.5 & 69.2 & & $2 \mathrm{H}-3,19-2 \mathrm{I}$ & 10.99 & 22.76 & 71.3 & 74.5 & 0.3 \\
\hline $1 \mathrm{H}-3,89-91$ & 3.89 & 27.20 & 63.0 & 58.2 & 0.5 & $2 \mathrm{H}-3,20-22$ & 11.00 & 21.79 & & & \\
\hline $1 \mathrm{H}-3,99-101$ & 3.99 & 13.67 & 60.5 & 31.7 & 0.3 & $2 \mathrm{H}-3,29-31$ & 11.09 & 15.32 & 42.7 & 61.6 & 0.2 \\
\hline $\mathrm{IH}-3,99-10 \mathrm{I}$ & 3.99 & & & & 0.3 & $2 \mathrm{H}-3,29-31$ & 11.09 & & 42.7 & & 0.2 \\
\hline $1 \mathrm{H}-3,109-111$ & 4.09 & 12.48 & 66.9 & 39.8 & 0.5 & $2 \mathrm{H}-3,39-41$ & 11.19 & 9.63 & 20.8 & 38.6 & 0.1 \\
\hline $1 \mathrm{H}-3,119-121$ & 4.19 & 22.02 & 56.8 & 42.0 & 0.4 & $2 \mathrm{H}-3,49-51$ & 11.29 & 23.61 & 43.9 & 35.2 & 0.4 \\
\hline $1 \mathrm{H}-3,129-131$ & 4.29 & 13.41 & 62.4 & 36.5 & 0.2 & $2 \mathrm{H}-3,59-61$ & 11.39 & 24.86 & 60.3 & 68.5 & 0.4 \\
\hline $1 \mathrm{H}-3,139-141$ & 4.39 & 11.45 & 74.8 & 27.5 & 0.2 & $2 \mathrm{H}-3,69-71$ & 11.49 & 25.72 & 60.0 & 63.0 & 0.4 \\
\hline IH $-3,148-150$ & 4.48 & 11.13 & 46.2 & 21.4 & 0.2 & $2 \mathrm{H}-3,79-81$ & 11.59 & 22.47 & 44.0 & 64.4 & 0.3 \\
\hline $1 \mathrm{H}-4,11-13$ & 4.61 & 12.85 & & & & $2 \mathrm{H}-3,89-91$ & 11.69 & 19.07 & 32.2 & 53.2 & 0.2 \\
\hline $1 \mathrm{H}-4,19-21$ & 4.69 & 15.84 & 45.5 & 31.5 & 0.2 & $2 \mathrm{H}-3,99-101$ & 11.79 & 23.72 & 54.3 & 60.3 & 0.3 \\
\hline $1 \mathrm{H}-4,29-31$ & 4.79 & 7.42 & 31.0 & 36.6 & 0.1 & $2 \mathrm{H}-3,109-111$ & 11.89 & 30.22 & 60.1 & 74.0 & 0.4 \\
\hline $1 \mathrm{H}-4,38-40$ & 4.88 & 19.23 & & & & $2 \mathrm{H}-3,119-121$ & 11.99 & 31.20 & 66.5 & 72.0 & 0.3 \\
\hline $1 \mathrm{H}-4,49-51$ & 4.99 & 23.54 & 55.3 & 53.0 & 0.6 & $2 \mathrm{H}-3,129-131$ & 12.09 & 13.57 & 51.2 & 44.6 & 0.2 \\
\hline $1 \mathrm{H}-4,59-61$ & 5.09 & 28.95 & 57.0 & 58.8 & 0.6 & $2 \mathrm{H}-3,139-141$ & 12.19 & 15.81 & 48.0 & 48.4 & 0.2 \\
\hline $1 \mathrm{H}-4,69-71$ & 5.19 & 22.92 & 51.5 & 52.7 & 0.5 & $2 \mathrm{H}-3,148-150$ & 12.28 & 11.72 & 39.4 & 46.5 & 0.2 \\
\hline $1 \mathrm{H}-4,89-91$ & 5.39 & 24.27 & 42.9 & 53.0 & 0.4 & $2 \mathrm{H}-4,9-11$ & 12.39 & 10.99 & 23.2 & 45.0 & 0.1 \\
\hline $1 \mathrm{H}-4,89-91$ & 5.39 & & 38.7 & 46.0 & & $2 \mathrm{H}-4,19-21$ & 12.49 & 15.18 & 37.7 & 52.2 & 0.3 \\
\hline $1 \mathrm{H}-4,99-101$ & 5.49 & 11.06 & 32.3 & 40.0 & 0.1 & $2 \mathrm{H}-4,29-31$ & 12.59 & 12.57 & 24.3 & 36.7 & 0.1 \\
\hline IH-4, $109-111$ & 5.59 & 12.57 & 36.5 & 30.0 & 0.1 & $2 \mathrm{H}-4,39-41$ & 12.69 & 27.38 & 54.4 & 50.4 & 0.5 \\
\hline IH-5,9-11 & 6.09 & 13.01 & 22.9 & 22.5 & 0.1 & $2 \mathrm{H}-4,39-41$ & 12.69 & & 54.4 & & 0.5 \\
\hline $1 \mathrm{H}-5,19-21$ & 6.19 & 21.20 & 53.3 & 29.2 & 0.2 & $2 \mathrm{H}-4,49-51$ & 12.79 & 27.84 & 52.5 & 64.1 & 0.5 \\
\hline $1 \mathrm{H}-5,29-31$ & 6.29 & 31.74 & 68.1 & 54.3 & 0.6 & $2 \mathrm{H}-4,59-61$ & 12.89 & 16.59 & 59.1 & 62.2 & 0.4 \\
\hline $1 \mathrm{H}-5,39-41$ & 6.39 & 30.92 & 64.9 & 62.8 & 0.4 & $2 \mathrm{H}-4,69-71$ & 12.99 & 18.88 & 55.2 & 55.5 & 0.2 \\
\hline
\end{tabular}




\begin{tabular}{|c|c|c|c|c|c|c|c|c|c|c|c|}
\hline \multirow[b]{2}{*}{$\begin{array}{l}\text { Core, section, } \\
\text { interval }(\mathrm{cm})\end{array}$} & \multirow[b]{2}{*}{$\begin{array}{l}\text { Depth* } \\
\text { (mbsf) }\end{array}$} & \multirow[b]{2}{*}{$\begin{array}{c}\% \text { Sand } \\
\text { fraction } \\
>63 \mu \mathrm{m} \\
(\%)\end{array}$} & \multicolumn{2}{|c|}{ Fragmentation } & \multirow{2}{*}{$\begin{array}{c}\text { Planktonic } \\
\text { Ratio } \\
\text { [\#SAC/ } \\
\text { (\#SAC+ } \\
\text { \#PUL+ } \\
\text { \#MENA+ } \\
\text { \#TUMI)] }\end{array}$} & \multirow[b]{2}{*}{$\begin{array}{l}\text { Core, section, } \\
\text { interval }(\mathrm{cm})\end{array}$} & \multirow[b]{2}{*}{$\begin{array}{l}\text { Depth* } \\
\text { (mbsf) }\end{array}$} & \multirow[b]{2}{*}{$\begin{array}{c}\% \text { Sand } \\
\text { fraction } \\
>63 \mu \mathrm{m} \\
(\%)\end{array}$} & \multicolumn{2}{|c|}{ Fragmentation } & \multirow{2}{*}{$\begin{array}{c}\text { Planktonic } \\
\text { Ratio } \\
\text { [\#SACl } \\
\text { (\#SAC+ } \\
\text { \#PUL+ } \\
\text { \#MENA+ } \\
\text { \#TUMI)] }\end{array}$} \\
\hline & & & $\begin{array}{c}\text { Whole } \\
\text { G. menardii } \\
(\%)\end{array}$ & $\begin{array}{c}\text { Whole } \\
\text { tests } \\
(\%)\end{array}$ & & & & & $\begin{array}{l}\text { Whole } \\
\text { G. menardii } \\
(\%)\end{array}$ & $\begin{array}{c}\text { Whole } \\
\text { tests } \\
(\%)\end{array}$ & \\
\hline $2 \mathrm{H}-4,79-81$ & 13.09 & 19.80 & 43.9 & 48.2 & 0.3 & $2 \mathrm{H}-6,148-150$ & 16.78 & 5.94 & 30.5 & 49.3 & 0.0 \\
\hline $2 \mathrm{H}-4,89-91$ & 13.19 & 15.86 & 46.5 & 57.3 & 0.4 & $2 \mathrm{H}-7,9-11$ & 16.89 & 5.89 & 25.0 & 40.1 & 0.0 \\
\hline $2 \mathrm{H}-4,99-101$ & 13.29 & 14.33 & 35.1 & 47.2 & 0.1 & $2 \mathrm{H}-7,19-21$ & 16.99 & 9.56 & 32.9 & 42.2 & 0.1 \\
\hline $2 \mathrm{H}-4,109-111$ & 13.39 & 18.57 & 36.5 & 39.7 & 0.2 & $2 \mathrm{H}-7,32-34$ & 17.12 & 24.39 & 41.1 & 59.3 & 0.2 \\
\hline $2 \mathrm{H}-4,119-121$ & 13.49 & 18.49 & 30.7 & 51.7 & 0.2 & $2 \mathrm{H}-7,39-41$ & 17.19 & 23.70 & 42.4 & 61.0 & 0.2 \\
\hline $2 \mathrm{H}-4,129-131$ & 13.59 & 17.79 & 38.9 & 50.4 & 0.2 & $2 \mathrm{H}-7,39-41$ & 17.19 & & & & 0.2 \\
\hline $2 \mathrm{H}-4,139-141$ & 13.69 & 11.53 & 25.6 & 36.3 & 0.1 & $2 \mathrm{H}-7,49-51$ & 17.29 & 13.37 & 39.5 & 42.6 & 0.1 \\
\hline $2 \mathrm{H}-4,139-141$ & 13.69 & & 21.8 & 36.5 & & $2 \mathrm{H}-7,53-55$ & 17.33 & 17.39 & & & \\
\hline $2 \mathrm{H}-5,9-11$ & 13.89 & 10.99 & 32.3 & 41.4 & 0.1 & $2 \mathrm{H}-7,59-61$ & 17.39 & 12.47 & 35.0 & 47.0 & 0.1 \\
\hline $2 \mathrm{H}-5,19-21$ & 13.99 & 17.22 & 30.9 & 36.7 & 0.2 & $2 \mathrm{H}-7,69-71$ & 17.49 & 17.10 & 25.6 & 49.2 & 0.1 \\
\hline $2 \mathrm{H}-5,29-31$ & 14.09 & 23.93 & 59.6 & 64.8 & 0.4 & $3 \mathrm{H}-1,9-11$ & 17.39 & 19.37 & 48.5 & 54.6 & \\
\hline $2 \mathrm{H}-5,39-41$ & 14.19 & 27.90 & 67.1 & 69.5 & 0.5 & $3 \mathrm{H}-1,19-21$ & 17.49 & 17.37 & 40.2 & 52.8 & \\
\hline $2 \mathrm{H}-5,49-51$ & 14.29 & 24.34 & 62.2 & 64.7 & 0.4 & $3 \mathrm{H}-1,29-31$ & 17.59 & 16.16 & 42.9 & 49.8 & \\
\hline $2 \mathrm{H}-5,59-61$ & 14.39 & 26.88 & 55.8 & 67.5 & 0.3 & $3 \mathrm{H}-1,39-41$ & 17.69 & 22.05 & 43.3 & 56.3 & \\
\hline $2 \mathrm{H}-5,69-71$ & 14.49 & 28.76 & 72.4 & 71.8 & 0.3 & $3 \mathrm{H}-1,49-51$ & 17.79 & 30.41 & 66.5 & 71.5 & \\
\hline $2 \mathrm{H}-5,69-71$ & 14.49 & & 67.0 & 67.7 & & $3 \mathrm{H}-\mathrm{I}, 59-61$ & 17.89 & 25.15 & 47.4 & 53.8 & \\
\hline $2 \mathrm{H}-5,79-81$ & 14.59 & 19.40 & 44.9 & 49.4 & 0.3 & $3 \mathrm{H}-1,69-71$ & 17.99 & 18.22 & 24.7 & 34.2 & \\
\hline $2 \mathrm{H}-5,89-91$ & 14.69 & 18.13 & 55.9 & 56.5 & 0.3 & $3 \mathrm{H}-1,79-81$ & 18.09 & 29.15 & 48.2 & 61.5 & \\
\hline $2 \mathrm{H}-5,99-101$ & 14.79 & 24.38 & 56.8 & 70.1 & 0.3 & $3 \mathrm{H}-1,89-91$ & 18.19 & 33.36 & 37.3 & 56.3 & \\
\hline $2 \mathrm{H}-5,109-111$ & 14.89 & 28.31 & 56.5 & 70.1 & 0.3 & $3 \mathrm{H}-1,99-101$ & 18.29 & 37.62 & & & \\
\hline $2 \mathrm{H}-5,119-12 \mathrm{I}$ & 14.99 & 31.62 & 49.2 & 69.2 & 0.3 & $3 \mathrm{H}-1,109-111$ & 18.39 & 39.95 & 69.6 & 75.1 & \\
\hline $2 \mathrm{H}-5,129-131$ & 15.09 & 16.19 & 59.7 & 60.8 & 0.2 & $3 \mathrm{H}-1,119-121$ & 18.49 & 28.67 & & & \\
\hline $2 \mathrm{H}-5,139-141$ & 15.19 & 19.17 & 43.2 & 42.0 & 0.1 & $3 \mathrm{H}-1,129-131$ & 18.59 & 26.31 & 72.2 & 73.5 & \\
\hline $2 \mathrm{H}-6,9-11$ & 15.39 & 3.63 & 34.9 & 31.3 & 0.1 & $3 \mathrm{H}-1,139-141$ & 18.69 & 21.44 & 53.1 & 62.3 & \\
\hline $2 \mathrm{H}-6,12-14$ & 15.42 & 8.98 & & & & $3 \mathrm{H}-2,9-11$ & 18.89 & 18.58 & 26.3 & 30.4 & \\
\hline $2 \mathrm{H}-6,19-21$ & 15.49 & 8.63 & 38.7 & 51.8 & 0.2 & $3 \mathrm{H}-2,19-21$ & 18.99 & 23.99 & 37.6 & 46.8 & \\
\hline $2 \mathrm{H}-6,21-23$ & 15.51 & 7.72 & & & & $3 \mathrm{H}-2,24-26$ & 19.04 & 24.97 & & & \\
\hline $2 \mathrm{H}-6,32-34$ & 15.62 & 7.75 & 21.8 & 33.3 & 0.0 & $3 \mathrm{H}-2,39-41$ & 19.19 & 36.63 & 59.0 & 59.7 & \\
\hline $2 \mathrm{H}-6,39-41$ & 15.69 & 10.38 & 26.0 & 29.4 & 0.0 & $3 \mathrm{H}-2,49-51$ & 19.29 & 33.91 & 58.1 & 71.3 & \\
\hline $2 \mathrm{H}-6,49-51$ & 15.79 & 12.06 & 62.9 & 60.7 & 0.1 & $3 \mathrm{H}-2,59-61$ & 19.39 & 30.35 & 62.1 & 72.7 & \\
\hline $2 \mathrm{H}-6,59-61$ & 15.89 & 14.37 & 44.7 & 58.8 & 0.4 & $3 \mathrm{H}-2,69-71$ & 19.49 & 19.95 & 44.0 & 50.2 & \\
\hline $2 \mathrm{H}-6,69-71$ & 15.99 & 16.69 & 26.1 & 56.7 & 0.6 & $3 \mathrm{H}-2,79-81$ & 19.59 & 12.35 & 24.4 & 45.7 & \\
\hline $2 \mathrm{H}-6,79-81$ & 16.09 & 14.29 & 36.2 & 58.3 & 0.4 & $3 \mathrm{H}-2,89-91$ & 19.69 & 13.17 & 22.8 & 36.2 & \\
\hline $2 \mathrm{H}-6,89-91$ & 16.19 & 5.31 & 41.2 & 51.3 & 0.0 & $3 \mathrm{H}-2,99-101$ & 19.79 & 22.39 & 45.5 & 45.4 & \\
\hline $2 \mathrm{H}-6,99-101$ & 16.29 & 7.31 & 40.7 & 41.6 & 0.1 & $3 \mathrm{H}-2,109-111$ & 19.89 & 34.61 & & & \\
\hline $2 \mathrm{H}-6,102-104$ & 16.32 & 7.01 & & & & $3 \mathrm{H}-2,119-121$ & 19.99 & 31.76 & 59.9 & 78.8 & \\
\hline $2 \mathrm{H}-6,109-111$ & 16.39 & 6.62 & 28.6 & 34.1 & 0.0 & $3 \mathrm{H}-2,129-131$ & 20.09 & 20.82 & & & \\
\hline $2 \mathrm{H}-6,119-121$ & 16.49 & 8.21 & 30.2 & 51.3 & 0.0 & $3 \mathrm{H}-2,139-141$ & 20.19 & 19.82 & 23.7 & 49.5 & \\
\hline $2 \mathrm{H}-6,129-131$ & 16.59 & 13.54 & 28.1 & 52.8 & 0.1 & $3 \mathrm{H}-2,148-150$ & 20.28 & 15.68 & 27.6 & 41.3 & \\
\hline $2 \mathrm{H}-6,139-141$ & 16.69 & 6.88 & 31.2 & 30.3 & 0.0 & & & & & & \\
\hline
\end{tabular}

*ODP depth assignment $=$ depth has not been adjusted.

$\doteqdot \mathrm{HSAC}=$ the number of whole tests of Globigerinoides sacculifer, \#PUL = the number of whole tests of Pulleniatina, \#MENA $=$ the number of whole tests of Globorotalia menardii, \#TUMI = the number of whole tests of Globorotalia tumida.

indicating that differences in response between species are not great. In what follows, we have combined these two indexes, giving whole tests (WT) twice the weight of whole $G$. menardii (Wmen), because the WT is statistically more significant by virtue of its greater sample population than Wmen. The resulting composite fragmentation index (CFI) is defined as follows:

$$
\mathrm{CFI}=\{(2[\mathrm{WT}]+[\mathrm{Wmen}]) / 3\},
$$

where square brackets denote variables in standard units (i.e., demeaned and divided by four times the standard deviation). The relationship between this index of fragmentation and the $\delta^{18} \mathrm{O}$ record of Pulleniatina (POX) is presented in Figure 4. CFI is positive when fragmentation is low. POX is plotted positive up to demonstrate the correspondence with the fragmentation record. As expected from previous work, glacial periods (labeled with even isotopic stage numbers) correlate with increased abundance of whole tests. Upon closer inspection, one can also see that the preservation record "lags" the isotope record (as noted by Moore et al., 1977) because preservation peaks occur at the end of the glacial intervals. This shift can be explained if it is assumed that deglaciation leads to a decrease in carbonate dissolution on the seafloor, and vice versa (Shackleton, 1977; Berger and Vincent, 1981). A decrease in productivity during deglaciation (e.g., Herguera and Berger, 1991) can produce such an effect (Keir and Berger, 1983).

To visualize the phase relationship between CFI and the change in the $\delta^{18} \mathrm{O}$ record, we compare CFI with a measure of the slope of the $\delta^{18} \mathrm{O}$ Pulleniatina (POX') record (Fig. 5). The derivative shown is one half of the $\delta^{18} \mathrm{O}$ difference between points $20 \mathrm{~cm}$ apart, calculated at an incremental spacing of $10 \mathrm{~cm}$. This simple method reduces the influence of spurious single points on the slope of the curve. The prominence of deglaciation events (labeled with the number of the preceding glaciation) is striking in this plot, supporting the asymmetric "saw-tooth" image of Broecker and van Donk (1970). There is a tendency for preservation peaks to approach the deglaciation peaks from below the level of maximum $\delta^{18} \mathrm{O}$ change. However, aside from this tendency for coincidence of peak values, some mismatches occur in the two curves. To optimize the fit between the preservation index and the two $\delta^{18} \mathrm{O}$ records (zero order and first derivative), we write an equation of the form:

$$
\mathrm{CFI}_{\mathrm{est}}=\left\{\left(k_{1}[\mathrm{POX}]\right)+\left(k_{2}\left[\mathrm{POX}^{\prime}\right]\right)\right\},
$$


Table 2. Productivity indexes, Hole 805C.

\begin{tabular}{|c|c|c|c|c|c|c|c|}
\hline $\begin{array}{l}\text { Core, section, } \\
\text { interval }(\mathrm{cm})\end{array}$ & $\begin{array}{l}\text { Depth* } \\
\text { (mbsf) }\end{array}$ & $\begin{array}{c}\text { Planktonic } \\
\text { ratio } \\
\text { [\#TUM/ } \\
(\# P U L+ \\
\text { \#TUM)]† }\end{array}$ & $\begin{array}{c}\text { Total } \\
\text { benthic } \\
\text { foraminifers } \\
(\# / g m)\end{array}$ & $\begin{array}{l}\text { Core, section, } \\
\text { interval }(\mathrm{cm})\end{array}$ & $\begin{array}{l}\text { Depth* } \\
\text { (mbsf) }\end{array}$ & $\begin{array}{c}\text { Planktonic } \\
\text { ratio } \\
\text { [\#TUM/ } \\
\text { (\#PUL + } \\
\text { \#TUM)]† }\end{array}$ & $\begin{array}{c}\text { Total } \\
\text { benthic } \\
\text { foraminifers } \\
\text { (\#/gm) }\end{array}$ \\
\hline IH-1,9-11 & 0.09 & & 32.5 & $1 \mathrm{H}-5,142-145$ & 7.42 & 0.8 & 13.2 \\
\hline $1 \mathrm{H}-1,13-15$ & 0.13 & 0.9 & 18.9 & IH-5, 149-151 & 7.49 & 0.7 & 14.7 \\
\hline $1 \mathrm{H}-1,19-21$ & 0.19 & & 25.7 & $2 \mathrm{H}-1,9-11$ & 7.89 & 0.7 & 19.4 \\
\hline $1 \mathrm{H}-1,21-23$ & 0.21 & 0.9 & 25.8 & $2 \mathrm{H}-1,19-21$ & 7.99 & 0.7 & 27.0 \\
\hline $1 \mathrm{H}-1,29-31$ & 0.29 & 0.8 & 27.1 & $2 \mathrm{H}-1,29-31$ & 8.09 & 0.7 & 23.5 \\
\hline $1 \mathrm{H}-1,39-41$ & 0.39 & 0.9 & 24.6 & $2 \mathrm{H}-1,39-41$ & 8.19 & 0.6 & 23.1 \\
\hline $1 \mathrm{H}-1,49-51$ & 0.49 & 0.8 & 39.3 & $2 \mathrm{H}-1,49-51$ & 8.29 & 0.8 & 21.1 \\
\hline $1 \mathrm{H}-1,59-61$ & 0.59 & 0.9 & 22.7 & $2 \mathrm{H}-1,49-51$ & 8.29 & 0.7 & \\
\hline $1 \mathrm{H}-1,69-71$ & 0.69 & 0.9 & 41.1 & $2 \mathrm{H}-1,59-61$ & 8.39 & 0.8 & 20.3 \\
\hline $1 \mathrm{H}-1,69-71$ & 0.69 & 0.9 & & $2 \mathrm{H}-1,69-71$ & 8.49 & 0.9 & 13.5 \\
\hline $1 \mathrm{H}-1,75-77$ & 0.75 & & 34.8 & $2 \mathrm{H}-1,79-81$ & 8.59 & 0.8 & 20.8 \\
\hline $1 \mathrm{H}-1,89-91$ & 0.89 & 0.9 & 36.3 & $2 \mathrm{H}-1,89-91$ & 8.69 & 0.7 & 19.3 \\
\hline IH-1, 89-91 & 0.89 & 0.9 & & $2 \mathrm{H}-1,99-101$ & 8.79 & 0.7 & 33.8 \\
\hline $1 \mathrm{H}-1,89-91$ & 0.89 & 0.9 & & $2 \mathrm{H}-1,109-111$ & 8.89 & 0.7 & 37.2 \\
\hline 1H-1,99-101 & 0.99 & 0.8 & 56.8 & $2 \mathrm{H}-1,119-121$ & 8.99 & 0.7 & 38.8 \\
\hline IH-1. 109-11] & 1.09 & 0.8 & 53.9 & $2 \mathrm{H}-1,129-131$ & 9.09 & 0.8 & 37.5 \\
\hline IH-1, 119-12। & 1.19 & 0.8 & 28.0 & $2 \mathrm{H}-1,139-141$ & 9.19 & 0.8 & 20.6 \\
\hline $1 \mathrm{H}-1,129-131$ & 1.29 & 1.0 & 15.5 & $2 \mathrm{H}-1,139-141$ & 9.19 & 0.8 & \\
\hline $1 \mathrm{H}-1,143-145$ & 1.43 & 0.8 & 31.4 & $2 \mathrm{H}-1,148-150$ & 9.28 & 0.8 & 19.7 \\
\hline $1 \mathrm{H}-2,4-6$ & 1.54 & 0.6 & 23.8 & $2 \mathrm{H}-2,9-11$ & 9.39 & 0.8 & 15.3 \\
\hline $1 \mathrm{H}-2,19-21$ & 1.69 & 0.8 & 31.1 & $2 \mathrm{H}-2,19-21$ & 9.49 & 0.8 & 16.2 \\
\hline $1 \mathrm{H}-2,29-31$ & 1.79 & 0.9 & 20.5 & $2 \mathrm{H}-2,29-31$ & 9.59 & 0.7 & 29.0 \\
\hline $1 \mathrm{H}-2,39-41$ & 1.89 & 0.6 & 39.5 & $2 \mathrm{H}-2,39-41$ & 9.69 & 0.7 & 38.0 \\
\hline $1 \mathrm{H}-2,49-51$ & 1.99 & 0.8 & 38.4 & $2 \mathrm{H}-2,49-51$ & 9.79 & 0.7 & 36.9 \\
\hline $1 \mathrm{H}-2,59-61$ & 2.09 & 0.8 & 46.6 & $2 \mathrm{H}-2,59-61$ & 9.89 & 0.7 & 32.3 \\
\hline $1 \mathrm{H}-2,69-71$ & 2.19 & 0.8 & 46.0 & $2 \mathrm{H}-2,69-71$ & 9.99 & 0.8 & 35.0 \\
\hline $1 \mathrm{H}-2,79-81$ & 2.29 & 0.8 & 61.1 & $2 \mathrm{H}-2,79-81$ & 10.09 & 0.8 & 29.7 \\
\hline $1 \mathrm{H}-2,89-91$ & 2.39 & 0.8 & 85.7 & $2 \mathrm{H}-2,89-91$ & 10.19 & 0.8 & 35.3 \\
\hline $1 \mathrm{H}-2,99-101$ & 2.49 & 0.8 & 55.9 & $2 \mathrm{H}-2,99-101$ & 10.29 & 0.8 & 34.6 \\
\hline $1 \mathrm{H}-2,109-111$ & 2.59 & 0.8 & 51.9 & $2 \mathrm{H}-2,109-111$ & 10.39 & 0.8 & 40.3 \\
\hline $1 \mathrm{H}-2,119-121$ & 2.69 & 0.8 & 70.0 & $2 \mathrm{H}-2,119-121$ & 10.49 & 0.8 & 36.3 \\
\hline $1 \mathrm{H}-2,129-131$ & 2.79 & 0.8 & 64.2 & $2 \mathrm{H}-2,129-131$ & 10.59 & 0.7 & 36.4 \\
\hline $1 \mathrm{H}-2,143-145$ & 2.93 & & 14.5 & $2 \mathrm{H}-2,139-141$ & 10.69 & 0.7 & 36.2 \\
\hline $1 \mathrm{H}-2,148-150$ & 2.98 & 0.7 & 19.1 & $2 \mathrm{H}-2,148-150$ & 10.78 & 0.7 & 43.8 \\
\hline $1 \mathrm{H}-3,9-11$ & 3.09 & 0.9 & 19.7 & $2 \mathrm{H}-3,9-11$ & 10.89 & 0.7 & 34.6 \\
\hline IH-3, 19-21 & 3.19 & 0.8 & 21.1 & $2 \mathrm{H}-3,19-21$ & 10.99 & 0.8 & \\
\hline IH-3, 29-31 & 3.29 & 0.8 & 29.8 & $2 \mathrm{H}-3,20-22$ & 11.00 & & 43.4 \\
\hline $1 \mathrm{H}-3,39-41$ & 3.39 & 0.9 & 21.2 & $2 \mathrm{H}-3,29-31$ & 11.09 & 0.7 & 32.0 \\
\hline $1 \mathrm{H}-3,49-51$ & 3.49 & 0.9 & 23.1 & $2 \mathrm{H}-3,29-31$ & 11.09 & 0.7 & \\
\hline $1 \mathrm{H}-3,59-61$ & 3.59 & & 36.5 & $2 \mathrm{H}-3,39-41$ & 11.19 & 0.7 & 21.7 \\
\hline $1 \mathrm{H}-3,69-71$ & 3.69 & 0.8 & 40.3 & $2 \mathrm{H}-3,49-51$ & 11.29 & 0.8 & 18.8 \\
\hline $1 \mathrm{H}-3,79-81$ & 3.79 & 0.8 & 37.4 & $2 \mathrm{H}-3,59-61$ & 11.39 & 0.8 & 7.0 \\
\hline $1 \mathrm{H}-3,89-91$ & 3.89 & 0.7 & 41.2 & $2 \mathrm{H}-3,69-71$ & 11.49 & 0.8 & 22.6 \\
\hline $1 \mathrm{H}-3,99-101$ & 3.99 & 0.7 & 46.7 & $2 \mathrm{H}-3,79-81$ & 11.59 & 0.8 & 29.2 \\
\hline $1 \mathrm{H}-3,99-101$ & 3.99 & 0.7 & & $2 \mathrm{H}-3,89-91$ & 11.69 & 0.7 & 39.5 \\
\hline $1 \mathrm{H}-3,109-111$ & 4.09 & 0.8 & 42.4 & $2 \mathrm{H}-3,99-101$ & 11.79 & 0.8 & 28.0 \\
\hline $1 \mathrm{H}-3,119-121$ & 4.19 & 0.9 & 17,0 & $2 \mathrm{H}-3,109-111$ & 11.89 & 0.9 & \\
\hline $1 \mathrm{H}-3,129-131$ & 4.29 & 0.7 & 24.3 & $2 \mathrm{H}-3,119-121$ & I1.99 & 0.8 & 27.3 \\
\hline $1 \mathrm{H}-3,139-141$ & 4.39 & 0.8 & 30.0 & $2 \mathrm{H}-3,129-131$ & 12.09 & 0.8 & 25.4 \\
\hline $1 \mathrm{H}-3,148-150$ & 4.48 & 0.8 & 21.6 & $2 \mathrm{H}-3,139-141$ & 12.19 & 0.8 & 36.7 \\
\hline $1 \mathrm{H}-4,11-13$ & 4.61 & & 18.3 & $2 \mathrm{H}-3,148-150$ & 12.28 & 0.7 & 17.9 \\
\hline $1 \mathrm{H}-4,19-21$ & 4.69 & 0.8 & 17.6 & $2 \mathrm{H}-4,9-11$ & 12.39 & 0.6 & 25.7 \\
\hline $1 \mathrm{H}-4,29-31$ & 4.79 & 0.8 & 16.1 & $2 \mathrm{H}-4,19-21$ & 12.49 & 0.7 & 20.3 \\
\hline $1 \mathrm{H}-4,38-40$ & 4.88 & & 20.7 & $2 \mathrm{H}-4,29-31$ & 12.59 & 0.7 & 18.5 \\
\hline $1 \mathrm{H}-4,49-51$ & 4.99 & 0.9 & & $2 \mathrm{H}-4,39-4 \mathrm{I}$ & 12.69 & 0.8 & 14.7 \\
\hline $1 \mathrm{H}-4,59-61$ & 5.09 & 0.9 & 22.5 & $2 \mathrm{H}-4,39-41$ & 12.69 & 0.7 & \\
\hline $1 \mathrm{H}-4,69-71$ & 5.19 & 0.8 & 37.1 & $2 \mathrm{H}-4,49-51$ & 12.79 & 0.8 & 18.1 \\
\hline $1 \mathrm{H}-4,89-91$ & 5.39 & 0.6 & 27.2 & $2 \mathrm{H}-4,59-61$ & 12.89 & 0.8 & 19.1 \\
\hline $1 \mathrm{H}-4,99-101$ & 5.49 & 0.6 & 29.4 & $2 \mathrm{H}-4,69-71$ & 12.99 & 0.7 & 42.4 \\
\hline $1 \mathrm{H}-4,109-111$ & 5.59 & 0.6 & 33.9 & $2 \mathrm{H}-4,79-8 \mathrm{I}$ & 13.09 & 0.6 & 56.2 \\
\hline $1 \mathrm{H}-5,9-11$ & 6.09 & 0.9 & 9.9 & $2 \mathrm{H}-4,89-91$ & 13.19 & 0.7 & 30.2 \\
\hline $1 \mathrm{H}-5,19-21$ & 6.19 & 0.9 & 12.3 & $2 \mathrm{H}-4,99-101$ & 13.29 & 0.8 & 32.9 \\
\hline $1 \mathrm{H}-5,29-31$ & 6.29 & 0.9 & 15.3 & $2 \mathrm{H}-4,109-111$ & 13.39 & 0.9 & 18.0 \\
\hline $1 \mathrm{H}-5,39-41$ & 6.39 & 0.8 & 11.6 & $2 \mathrm{H}-4,119-121$ & 13.49 & 0.9 & 26.0 \\
\hline $1 \mathrm{H}-5,49-51$ & 6.49 & 0.8 & 21.2 & $2 \mathrm{H}-4,129-131$ & 13.59 & 0.8 & 19.3 \\
\hline $1 \mathrm{H}-5,49-51$ & 6.49 & 0.7 & & $2 \mathrm{H}-4,139-141$ & 13.69 & 0.8 & 20.3 \\
\hline $1 \mathrm{H}-5,59-61$ & 6.59 & 0.7 & 37.3 & $2 \mathrm{H}-5,9-11$ & 13.89 & 0.8 & 15.3 \\
\hline $1 \mathrm{H}-5,69-71$ & 6.69 & 0.6 & 31.6 & $2 \mathrm{H}-5,19-21$ & 13.99 & 0.9 & 18.1 \\
\hline $1 \mathrm{H}-5,79-81$ & 6.79 & 0.8 & 28.9 & $2 \mathrm{H}-5,29-31$ & 14.09 & 0.9 & 14.6 \\
\hline $1 \mathrm{H}-5,89-91$ & 6.89 & 0.7 & 25.2 & $2 \mathrm{H}-5,39-41$ & 14.19 & 0.7 & 30.5 \\
\hline 1H-5, 99-101 & 6.99 & 0.8 & 22.6 & $2 \mathrm{H}-5,49-51$ & 14.29 & 0.7 & 36.9 \\
\hline $1 \mathrm{H}-5,109-111$ & 7.09 & 0.7 & 32.3 & $2 \mathrm{H}-5,59-61$ & 14.39 & 0.6 & 28.4 \\
\hline IH-5, 119-121 & 7.19 & 0.8 & 23.1 & $2 \mathrm{H}-5,69-7 \mathrm{I}$ & 14.49 & 0.7 & 35.2 \\
\hline $1 \mathrm{H}-5,129-131$ & 7.29 & 0.7 & 24.3 & $2 \mathrm{H}-5,79-8 \mathrm{I}$ & 14.59 & 0.8 & 36.7 \\
\hline
\end{tabular}


Table 2 (continued).

\begin{tabular}{|c|c|c|c|c|c|c|c|}
\hline $\begin{array}{l}\text { Core, section, } \\
\text { interval }(\mathrm{cm})\end{array}$ & $\begin{array}{l}\text { Depth* } \\
\text { (mbsf) }\end{array}$ & $\begin{array}{c}\text { Planktonic } \\
\text { ratio } \\
\text { [\#TUM/ } \\
\text { (\#PUL + } \\
\text { \#TUM)]† }\end{array}$ & $\begin{array}{c}\text { Total } \\
\text { benthic } \\
\text { foraminifers } \\
(\# / g m)\end{array}$ & $\begin{array}{l}\text { Core, section, } \\
\text { interval }(\mathrm{cm})\end{array}$ & $\begin{array}{l}\text { Depth* } \\
\text { (mbsf) }\end{array}$ & $\begin{array}{c}\text { Planktonic } \\
\text { ratio } \\
\text { [\#TUM/ } \\
\text { (\#PUL + } \\
\text { \#TUM)]† }\end{array}$ & $\begin{array}{c}\text { Total } \\
\text { benthic } \\
\text { foraminifers } \\
(\# / g m)\end{array}$ \\
\hline $2 \mathrm{H}-5,89-91$ & 14.69 & 0.8 & 35.0 & $2 \mathrm{H}-7,69-71$ & 17.49 & 0.5 & 60.3 \\
\hline $2 \mathrm{H}-5,99-101$ & 14.79 & 0.8 & 23.6 & $3 \mathrm{H}-1,9-11$ & 17.39 & & 18.0 \\
\hline $2 \mathrm{H}-5,109-111$ & 14.89 & 0.9 & 23.3 & $3 \mathrm{H}-1,19-21$ & 17.49 & & 37.7 \\
\hline $2 \mathrm{H}-5,119-121$ & 14.99 & 0.9 & 29.9 & $3 \mathrm{H}-1,29-31$ & 17.59 & & 29.1 \\
\hline $2 \mathrm{H}-5,129-131$ & 15.09 & 0.9 & 36.8 & $3 \mathrm{H}-1,39-41$ & 17.69 & & 42.8 \\
\hline $2 \mathrm{H}-5,139-141$ & 15.19 & 0.8 & 57.2 & $3 \mathrm{H}-1,49-51$ & 17.79 & & 34.9 \\
\hline $2 \mathrm{H}-6,9-11$ & 15.39 & 0.4 & & $3 \mathrm{H}-1,59-61$ & 17.89 & & 41.3 \\
\hline $2 \mathrm{H}-6,12-14$ & 15.42 & & 48.1 & $3 \mathrm{H}-1,69-71$ & 17.99 & & 23.2 \\
\hline $2 \mathrm{H}-6,19-21$ & 15.49 & 0.5 & & $3 \mathrm{H}-1,79-81$ & 18.09 & & 15.2 \\
\hline $2 \mathrm{H}-6,21-23$ & 15.51 & & 28.7 & $3 \mathrm{H}-1,89-91$ & 18.19 & & 24.8 \\
\hline $2 \mathrm{H}-6,32-34$ & 15.62 & 0.6 & 18.9 & $3 \mathrm{H}-1,99-101$ & 18.29 & & 27.5 \\
\hline $2 \mathrm{H}-6,39-41$ & 15.69 & 0.7 & 22.7 & $3 \mathrm{H}-1,109-111$ & 18.39 & & 26.0 \\
\hline $2 \mathrm{H}-6,49-51$ & 15.79 & 0.9 & 13.1 & $3 \mathrm{H}-1,119-121$ & 18.49 & & 28.2 \\
\hline $2 \mathrm{H}-6,59-61$ & 15.89 & 0.9 & 13.7 & $3 \mathrm{H}-1,129-131$ & 18.59 & & 30.2 \\
\hline $2 \mathrm{H}-6,69-71$ & 15.99 & 0.8 & 20.1 & $3 \mathrm{H}-1,139-141$ & 18.69 & & 29.1 \\
\hline $2 \mathrm{H}-6,79-81$ & 16.09 & 0.8 & 31.1 & $3 \mathrm{H}-2,9-11$ & 18.89 & & 33.9 \\
\hline $2 \mathrm{H}-6,89-91$ & 16.19 & 0.6 & 36.6 & $3 \mathrm{H}-2,19-21$ & 18.99 & & 28.4 \\
\hline $2 \mathrm{H}-6,99-101$ & 16.29 & 0.5 & & $3 \mathrm{H}-2,24-26$ & 19.04 & & 33.2 \\
\hline $2 \mathrm{H}-6,109-111$ & 16.39 & 0.5 & 42.1 & $3 \mathrm{H}-2,39-41$ & 19.19 & & 24.1 \\
\hline $2 \mathrm{H}-6,119-121$ & 16.49 & 0.7 & 31.8 & $3 \mathrm{H}-2,49-5 \mathrm{I}$ & 19.29 & & 30.9 \\
\hline $2 \mathrm{H}-6,129-131$ & 16.59 & 0.8 & 22.6 & $3 \mathrm{H}-2,59-61$ & 19.39 & & 51.6 \\
\hline $2 \mathrm{H}-6,139-141$ & 16.69 & 0.8 & 22.6 & $3 \mathrm{H}-2,69-71$ & 19.49 & & 54.1 \\
\hline $2 \mathrm{H}-6,148-150$ & 16.78 & 0.6 & 26.6 & $3 \mathrm{H}-2,79-81$ & 19.59 & & 25.6 \\
\hline $2 \mathrm{H}-7,9-11$ & 16.89 & 0.5 & 30.6 & $3 \mathrm{H}-2,89-91$ & 19.69 & & \\
\hline $2 \mathrm{H}-7,19-21$ & 16.99 & 0.7 & 15.1 & $3 \mathrm{H}-2,99-101$ & 19.79 & & 27.7 \\
\hline $2 \mathrm{H}-7,32-34$ & 17.12 & 0.8 & 23.1 & $3 \mathrm{H}-2,109-111$ & 19.89 & & \\
\hline $2 \mathrm{H}-7,39-41$ & 17.19 & 0.8 & 28.0 & $3 \mathrm{H}-2,119-121$ & 19.99 & & 35.5 \\
\hline $2 \mathrm{H}-7,39-41$ & 17.19 & 0.8 & & $3 \mathrm{H}-2,129-131$ & 20.09 & & 32.4 \\
\hline $2 \mathrm{H}-7,49-51$ & 17.29 & 0.7 & & $3 \mathrm{H}-2,139-141$ & 20.19 & & 43.7 \\
\hline $2 \mathrm{H}-7,53-55$ & 17.33 & & 85.2 & $3 \mathrm{H}-2,148-150$ & 20.28 & & 52.2 \\
\hline $2 \mathrm{H}-7,59-61$ & 17.39 & 0.4 & 48.5 & & & & \\
\hline
\end{tabular}

*ODP depth assignment $=$ depth has not been adjusted.

†TUM = the number of whole tests of Globorotalia tumida, and \#PUL = the number of whole tests of Pulleniatina.

where the variables are as described and square brackets denote standardization. Requiring that the sum of squares of differences is minimized, and that the two coefficients add to unity, we obtain $k_{1}=0.6$ and $k_{2}=0.4$.

One can see that the $\mathrm{CFI}_{\text {est }}$ resulting from Equation (2) fits the original curve quite well over most of the record (Fig. 6), demonstrating that both the state of the ocean climate and its rate of change are important for the preservation of carbonate on the deep-sea floor. We also see from this exercise that preservation is particularly poor in the vicinity of Isotope Stage 13 (ca. 450-500 k.y. ago) and is particularly good for Stage 16 (ca. 600-650 ka), if we assume that preservation changes are driven by changes in $[\mathrm{POX}]$ and $\left[\mathrm{POX}^{\prime}\right]$. Furthermore, fluctuations in preservation are rather large in the lower portion of the record below $17 \mathrm{mbsf}$, where obliquity cycles dominate. These changes in the quality of the match suggest that the sensitivity of the preservation record to climatic forcing changes through time.

\section{Sand Content}

The sand content of deep-sea carbonates decreases as dissolution progresses on the Ontong Java Plateau (Johnson et al., 1977; Wu et al., 1991) and elsewhere. The reason is that the shells of foraminifers are weakened by partial dissolution and disintegrate both on the seafloor and during sample preparation. Thus, material moves from the coarse fraction into the finer fractions. Inspection of the fine sand in samples taken from greater depths on the seafloor, for example, reveals the presence of an increasing amount of small fragments originally belonging to larger shells. If this process is dominant, the whole-test index (CFI) should show good agreement with the percent sand record (SAND index). This is indeed the case over most of the record (Fig. 7). The correspondence is good to excellent for the time before Isotope Stage 8 (ca. 250 k.y.). In the uppermost portion, however, the sand content is much higher than expected from the abundance of whole tests. Thus, preservational effects alone cannot explain most of the sand record in the last $250 \mathrm{k} . \mathrm{y}$. Other mechanisms producing variations, such as large changes in winnowing or bioturbation, must be invoked. It is not clear why the last 250 k.y. should be so different from the previous million years on this account. Apparently, winnowing activities greatly increased in this region in the latest Quaternary. If this change in dynamics is a general phenomenon, the common practice of using the history of the last $150 \mathrm{k.y}$. as a model for the previous million years must be viewed with caution.

\section{Abundance of G. sacculifer}

Tests of the species $G$. sacculifer are generally more susceptible to the effects of dissolution than the less porous tests of Pulleniatina, $G$. menardii, or $G$. tumida. All of these taxa are abundant in the western equatorial Pacific, so that their relative abundance is readily determined. Although this ratio may be influenced by climatic factors as well as preservational ones, Site 805 is deep enough that dissolution might be expected to deliver a strong overprint over the original ratios in the supply. The index we use (SAC index) is the percentage of whole $G$. sacculifer divided by the sum of whole $G$. sacculifer, whole Pulleniatina, whole $G$. menardii, and whole $G$. tumida (to dampen the effect of outliers in the ratio). Our count, as mentioned, only involves the upper two cores (Cores 130-805C-1H and -2H) to a depth of $17 \mathrm{mbsf}$ (corrected). The correspondence of the SAC index with the CFI is quite good (Fig. 8) and bears out the expectation based on earlier work (Parker and Berger, 1971). Significant mismatches occur at Isotope Stages 8, 10, and 20 (G. sacculifer being much more 


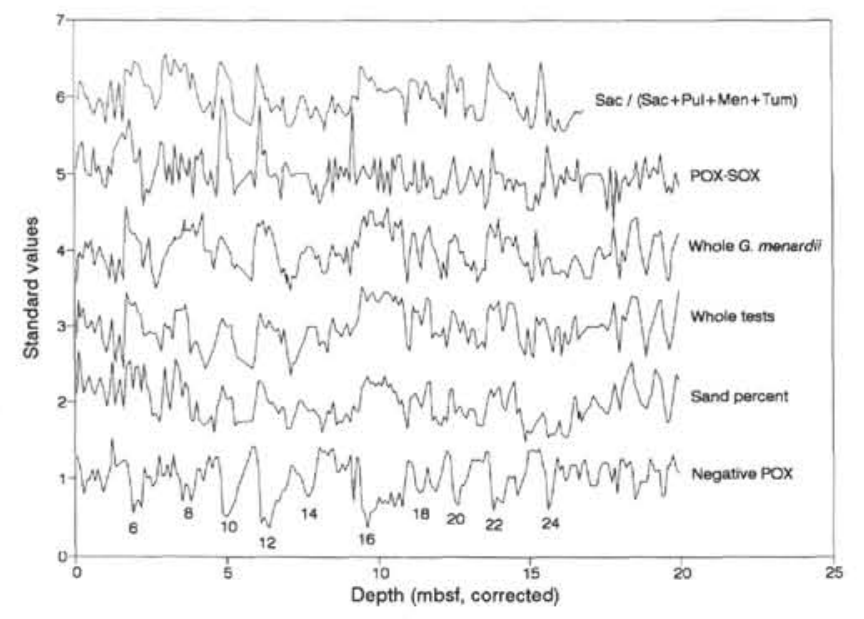

Figure 2. Preservation indexes and $\delta^{18} \mathrm{O}$ for Hole $805 \mathrm{C}$ vs. corrected depth. The $\delta^{18} \mathrm{O}$ record is given for reference. All fields in this figure and following figures are standardized according to $(x-A V G) /[(4)$ (STD) $]$. Positive values indicate good preservation. Fields from the bottom up are negative $\delta^{18} \mathrm{O}$ (Pulleniatina); sand fraction ( $w t \%>63 \mathrm{~mm}$ ); percent abundance of all whole planktonic foraminiferal tests relative to total number of particles in size fraction $250-425 \mu \mathrm{m}$; percent abundance of whole tests $G$. menardii complex relative to total number of particles in size fraction $250-425 \mu \mathrm{m} ; \Delta \delta^{18} \mathrm{O}$ (Pulleniatina minus G. sacculifer $)$; and number of tests of $[(G$. sacculifer $) /(G$. sacculifer + Pulleniatina $+G$. menardii $+G$. tumida $)$ ]. Isotopic stage numbers are given for reference in this figure and following figures.

abundant relative to Pulleniatina, G. menardii, and $G$. tumida than expected) and at Stage 16 (showing lower than expected abundance of $G$. sacculifer).

\section{Summary}

We have shown that the various preservation indexes (whole tests of foraminifers, sand content, and species abundance) correlate in the expected fashion, although mismatches and discrepancies exist in places. Generally, glacial intervals, as identified by the $\delta^{18} \mathrm{O}$ record,

Table 3. Age-depth conversion based on 41-k.y. $\delta^{18} \mathrm{O}$ obliquity cycle, Hole $805 \mathrm{C}$.

\begin{tabular}{crrr}
\hline $\begin{array}{c}\text { Adjusted } \\
\text { depth } \\
(\mathrm{m})\end{array}$ & $\begin{array}{r}\text { Age } \\
(\mathrm{k} . \mathrm{y} .)\end{array}$ & $\begin{array}{r}\text { Age }^{\mathrm{b}} \\
(\mathrm{k} . \mathrm{y} .)\end{array}$ & $\begin{array}{c}\Delta \text { Age }^{\mathrm{a} . \mathrm{b}} \\
\text { (k.y.) }\end{array}$ \\
\hline 0 & 0 & 0 & 0 \\
1 & 65 & 72 & -7 \\
2 & 137 & 148 & -11 \\
3 & 204 & 225 & -21 \\
4 & 281 & 285 & -4 \\
5 & 346 & 351 & -5 \\
6 & 411 & 414 & -3 \\
7 & 474 & 477 & -3 \\
8 & 527 & 534 & -7 \\
9 & 590 & 593 & -3 \\
10 & 663 & 655 & 8 \\
11 & 733 & 704 & 29 \\
12 & 799 & 754 & 45 \\
13 & 861 & 818 & 43 \\
14 & 920 & 881 & 39 \\
15 & 969 & 931 & 38 \\
16 & 1026 & 983 & 43 \\
17 & 1078 & 1039 & 39 \\
18 & 1131 & 1091 & 40 \\
19 & 1185 & 1144 & 41 \\
& & &
\end{tabular}

a This study (Pulleniatina).

${ }^{b}$ Berger et al. (this volume, G. sacculifer and Pulleniatina).

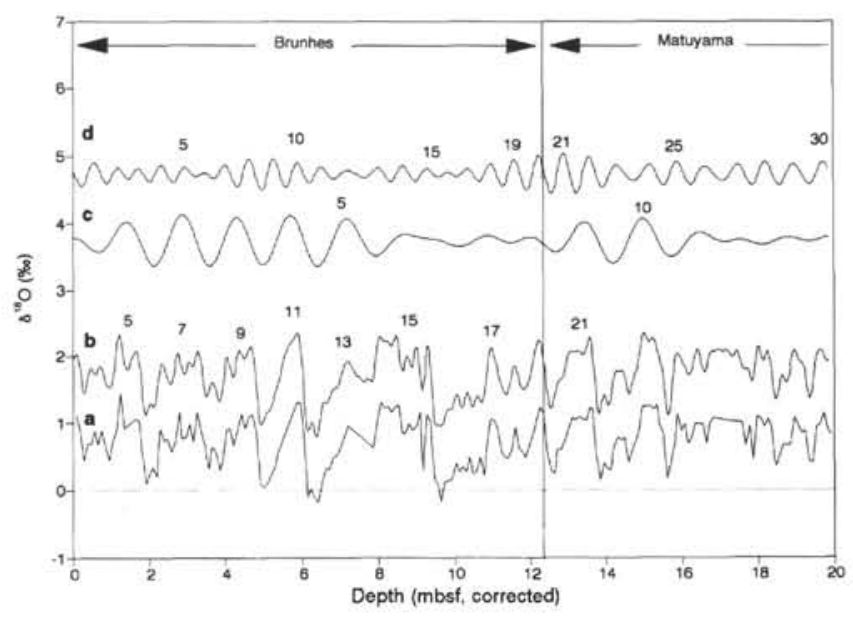

Figure 3. Obliquity and eccentricity components of Hole $805 \mathrm{C} \delta^{18} \mathrm{O}$ signal Pulleniatina; and $\delta^{18} \mathrm{O}$ Pulleniatina vs. corrected depth. Fields from the bottom up are (a) negative $\delta^{18} \mathrm{O}$ Pulleniatina; (b) reconstructed negative $\delta^{18} \mathrm{O}$ using harmonics 1-80 (vertical offset: +1); (c) 100-k.y. eccentricity component using harmonics 10-15 (vertical offset: +3 ); and (d) 41-k.y. obliquity component using harmonics $25-35$ (vertical offset: +4 ).

are characterized by good preservation, whereas the reverse is true for interglacials. In addition, the rate of change and its direction are important: deglaciation is associated with enhanced preservation, reglaciation is associated with increased dissolution. Combining all of these indexes into one,

$$
\mathrm{CDI}=\{0.5[\mathrm{CFI}]+0.25[\mathrm{SAND}]+0.25[\mathrm{SAC}]\},
$$

yields a composite dissolution index (CDI) that corresponds very well with the $\delta^{18} \mathrm{O}$ record (Fig. 9). In the fashion described in Equation (2), the best fit of $\mathrm{CDI}_{\text {est }}$ is obtained by setting the coefficients $k_{1}$ and $k_{2}$ to 0.55 and 0.45 , respectively,

$$
\mathrm{CDI}_{\text {est }}=\left\{\left(k_{1}[\mathrm{POX}]\right)+\left(k_{2}\left[\mathrm{POX}^{\prime}\right]\right)\right\},
$$

indicating that the state of the system is roughly equal in importance to the rate of change. Additional information is necessary to determine

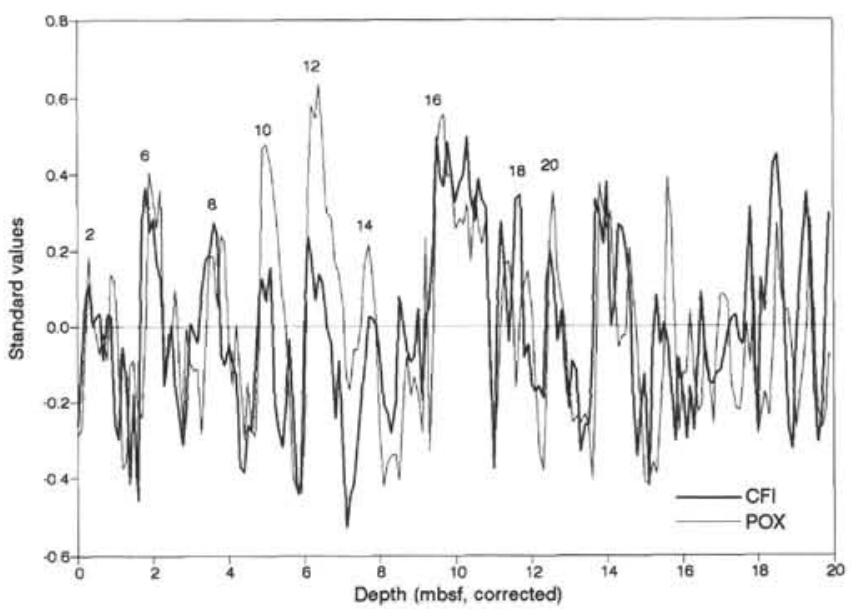

Figure 4. Standardized composite fragmentation index (CFI) and $\delta^{18} \mathrm{O}$ Pulleniatina $(\mathrm{POX})$ vs. corrected depth. $\mathrm{CFI}=(0.66)$ (standardized whole test index $)+(0.33)$ (standardized $G$. menardii index). Positive CFI values indicate good preservation. 


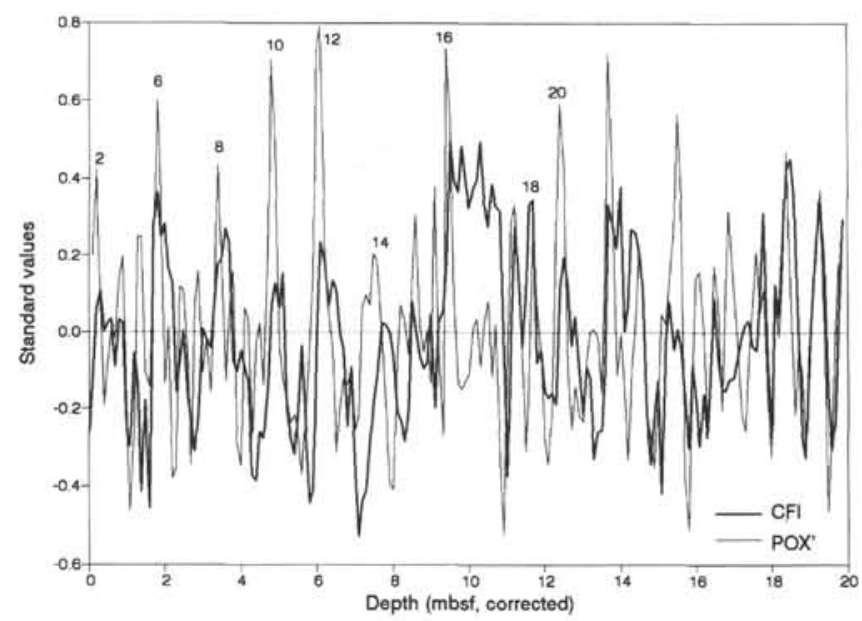

Figure 5. Standardized local change of $\delta^{18} \mathrm{O}$ Pulleniatina $\left(\mathrm{POX}^{\prime}\right)$ and $\mathrm{CFI}$ vs. corrected depth. The local change of $\delta^{18} \mathrm{O}$ is one half of the difference between $\delta^{18} \mathrm{O}$ values spaced $20 \mathrm{~cm}$ apart calculated at a $10-\mathrm{cm}$ spacing. Evenly spaced data points were produced by interpolation. In essence, this is a quick approximation of the first derivative of the $\delta^{18} \mathrm{O}$ curve, which reduces the effect of spurious single values. Positive CFI values indicate good preservation.

just how state and rate of change are to be defined in terms of oceanography. We suggest that three factors will turn out to be important as the internal driving variables of carbonate preservation: (1) the intensity of the NADW production, which governs the asymmetry in carbonate accumulation between the Atlantic and Pacific (Olausson, 1965; Berger, 1970b); (2) the extent to which shelves trap or release carbonate (Berger and Winterer, 1974; Milliman, 1974); and (3) the productivity level of the ocean, and the partitioning of productivity between coastal and open ocean (Keir and Berger, 1983; Berger and Keir, 1984).

\section{Productivity}

\section{G. tumida vs. Pulleniatina}

The abundance of G. tumida relative to the sum of Pulleniatina and $G$. tumida is a measure of the degree of upwelling along the equator in the Pacific. The relative dominance of key species changes from east to west along the equator from Globoquadrina dutertrei, to Globorotalia tumida, and to Pulleniatina, when this relationship is not obscured by dominant species in well-preserved samples. Parker and Berger (1971) suggest that this east-west gradient should be useful in paleogeographic reconstruction of the westward extent of the high fertility zone that originates in the eastern tropical Pacific. The ratio of $G$. tumida to the sum of Pulleniatina and $G$. tumida (TUM index; Table 2), does indeed show the expected relationship to glacial-interglacial fluctuations in $\delta^{18} \mathrm{O}$, with increased abundance of G. tumida during glacials (Fig. 10). The match is poor, however, between Stages 14 and 17 (ca. 500-700 ka). Also, for the last 200 k.y., the match is not good. Amplitudes of the TUM signal are uncommonly large from 15 mbsf downward, where obliquity cycles begin to dominate.

\section{Benthic Foraminifer Abundance}

The accumulation rate of benthic foraminifers in the sediments of the Ontong Java Plateau can be used to reconstruct glacial-to-interglacial changes in productivity (Herguera and Berger, 1991). Unfortunately, accumulation rates are not easily obtained on the relevant scale of variation because instantaneous accumulation rates are sensitive to small errors in successive age assignments. Here we use the total number of benthic foraminifers per gram of dry sediment (BF/g

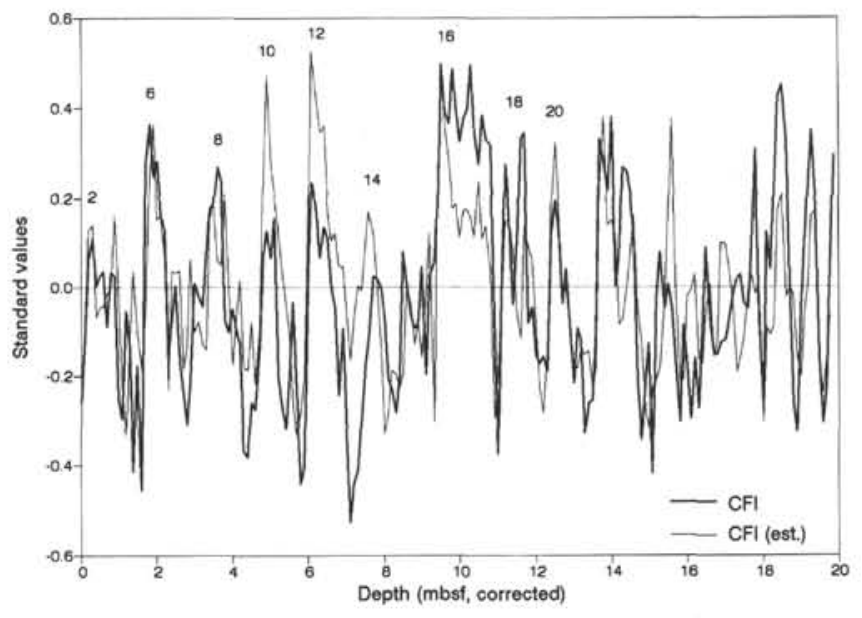

Figure 6. Standardized CFI (estimated) and standardized CFI vs. corrected depth. CFI (est.) $=(0.6)\left(\delta^{18} \mathrm{O}\right)+(0.4)\left(\delta^{18} \mathrm{O}^{\prime}\right)$. Positive values indicate good preservation.

index) (Table 2) to track changes in productivity (in fact assuming that sedimentation rates do not vary much downcore). As expected from previous studies (Herguera and Berger, 1991; Burke et al., in press), the abundance of total benthic foraminifers is higher during glacial intervals than during interglacials (Fig. 11). Benthic foraminifers can be concentrated by the effects of differential dissolution, in poorly preserved samples (Parker and Berger, 1971). However, the generally higher abundance of benthic foraminifers during glacials in association with better preservation suggests that benthic abundance is not significantly affected by dissolution in the record at hand. It seems unlikely that higher fluxes of organic matter associated with increased productivity would be sufficient to concentrate the dissolution-resistant benthic foraminifers because foraminifers are better preserved during glacials when benthic foraminifers are more abundant. Qualitatively, the match is quite good, the coincidence of phase being remarkable back to about $1 \mathrm{Ma}$ (ca. $16 \mathrm{mbsf}$ ). In detail, mismatches in amplitude are common. Stage 6 shows abundances that are much too great, whereas Stages $10,12,14$, and 16 show much reduced abundances of benthic foraminifers. Taking the changes in accumulation rate into account (as deduced from counting obliquity cycles; see below) could ameliorate these mismatches. However, this seems unlikely for the lower part of the section. Because of the uncertainty surrounding sedimentation rate determination for short intervals (cf. Berger et al., this volume), we have chosen not to calculate accumulation rates for benthic foraminifers in this report.

\section{Comparisons of Indexes}

The correspondence between the two productivity indexes TUM and $\mathrm{BF} / \mathrm{g}$ is only moderately good; indeed, striking mismatches are evident in places (Fig. 12). The most glaring example is the period around late Stage 7 , where the $\mathrm{BF} / \mathrm{g}$ index exhibits a remarkable excursion that is in no way reflected in the TUM record. The period after that time shows large positive discrepancies of the BF/g values as well. Earlier we noted the unusually high sand content in these sediments. Winnowing should affect BF/g but not TUM. Also, differential dissolution effects must be considered.

At this juncture, we have no reason to prefer one index over the other. Thus, we combine them into one productivity index, CPI (being the mean of two standardized productivity values, standardized), and compare this index with the original $\delta^{18} \mathrm{O}$ record (Fig. 13). The correspondence is moderately good, with the mismatches noted earlier persisting. 


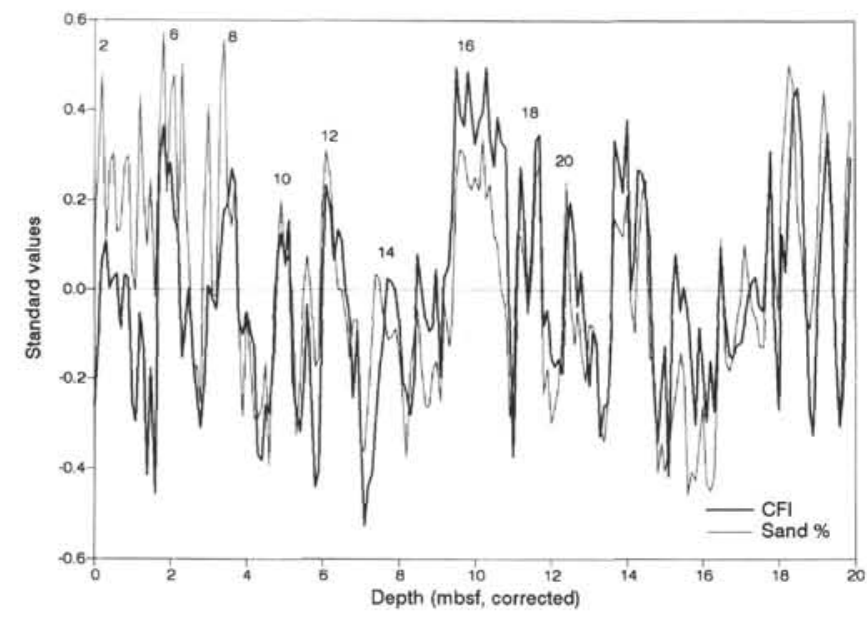

Figure 7. Standardized CFI and standardized percent sand vs. corrected depth. Percent sand is the dry weight percent of particles $>63 \mu \mathrm{m}$. Positive values indicate good preservation.

It has been suggested repeatedly that carbonate dissolution depends to some degree on the supply of organic matter to the seafloor (e.g., Berger, 1970b; Emerson and Bender, 1981; Archer, 1991). If this is important in our sample set, we should find a strong negative correlation between productivity and preservation. Comparing the two available indexes (CPI and CDI), we find that they are in fact positively correlated over much of the record (Fig. 14). However, this correspondence is much weaker than that of the CDI with the $\delta^{18} \mathrm{O}$ record. In fact, adding CPI as a third factor into an equation such as Equation (4) yields no improvement of the estimate of the intensity of dissolution. Thus, whatever contribution productivity (as measured by our indexes) makes to the variability of preservation, it is already contained in the fluctuations of the $\delta^{18} \mathrm{O}$ values of Pulleniatina. Although these observations do not rule out productivity effects on preservation, they suggest that productivity plays a subordinate role (leaving bottom-water saturation as the chief controlling factor).

\section{DISCUSSION}

\section{Comparison with Hole 806B}

It has long been realized that the pelagic $\delta^{18} \mathrm{O}$ record is altered by differential dissolution (reviewed in Wu and Berger, 1991). The sense of this effect is such as to enrich an assemblage in ${ }^{18} \mathrm{O}$-rich individuals by removal of the more open-structured specimens that made their shells in warm, shallow water. This effect will automatically ensure some correlation between the record of dissolution and that of $\delta^{18} \mathrm{O}$, which should oppose the normal glacial-interglacial relationship $\left(k_{1}\right.$ in Eq. 4) but reinforce the one tied to change ( $k_{2}$ in Eq. 4). To obtain the magnitude of the effect, one has to compare two records taken at different depths along the flanks of the plateau, as did Shackleton and Opdyke (1976). The shallower of the two records can serve as a standard against which to measure the deviation in the deeper one, a difference that can then be ascribed to the effects of differential dissolution. Unfortunately, when matching the cores based on their dissolution-susceptible signals, phase information tends to be lost or distorted (Wu et al., 1990).

The first step in preparing a comparison of two cores is to adjust the depth of one core in synchroneity with that of the other by finding the sedimentation rate ratios (SRRs) between the two cores. We use the percent sand records in Holes 805C (Table 1) and 806B (Table 4) to make the necessary correlation (Fig. 15). A first crude visual fit is achieved by applying the SRR coefficients $1.41,1.05$, and 1.15 to the depth segments of Hole $805 \mathrm{C}$ shown in the Figure 15. The presence of a coring gap of about $60 \mathrm{~cm}$ then becomes evident in Hole 806B.

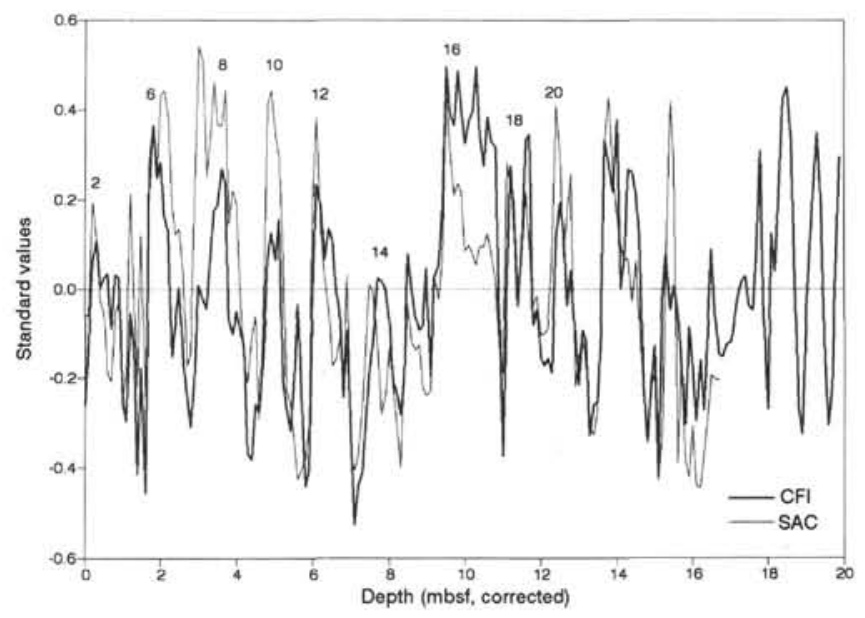

Figure 8. Standardized CFI and standardized abundance of dissolution susceptible species (SAC) vs. corrected depth. The SAC ratio used here is the number of whole tests of $G$. sacculifer/ $(G$. sacculifer + Pulleniatina $+G$. tumida $+G$. menardii). Positive values indicate good preservation.

A similar gap was reported by Berger et al. (this volume) based on matching isotopes and on a visual check using GRAPE data. The percent sand values for Hole $805 \mathrm{C}$ are seen to be generally (but not invariably) lower than the corresponding values in Hole 806B, as expected if sand content tracks dissolution history. Peak sand content in Hole $806 \mathrm{~B}$ is associated with glacial stages and is higher in many instances relative to Hole $805 \mathrm{C}$. Periods of increased winnowing during glacial time (Wu and Berger, 1991) should exhibit amplified differences between percent sand at these sites as fines removed from shallower sites are transported to deeper sites. In this context, increased bioturbation (bio-resuspension) from increased productivity or an increase in deep-sea tidal energy, or both, may be considered as possible causes of winnowing.

The sand-based crude match results in a remarkably good fit of negative $G$. sacculifer $\delta^{18} \mathrm{O}$ values (Fig. 16). On the whole, $\delta^{18} \mathrm{O}$ values in Hole $806 \mathrm{~B}$ tend to be slightly more negative than those in Hole $805 \mathrm{C}$, as expected based on the effects of differential dissolution. Within the species $G$. sacculifer, specimens living in shallow water tend to be thin walled and highly porous. Thick-walled terminal morphologies are produced in deeper and colder waters. With dissolution, the selective removal of isotopically light variants of $G$. sacculifer occurs. The effect is expressed preferentially during the interglacials (labeled with their isotope stages), confirming the observation that dissolution is more important during these intervals. The magnitude of the effect is typically $<0.2 \%$, but it can reach up to about $0.3 \%$. One important result of the $\delta^{18} \mathrm{O}$ match is that the Hole $805 \mathrm{C}$ record remains trustworthy as a guide to the state of the system, despite interference from dissolution. However, between isotopic Stages 10 and 18 , the Hole $805 \mathrm{C}$ Pulleniatina $\delta^{18} \mathrm{O}$ record is also offset toward heavier values during glacials. This suggests that a process other than dissolution may be affecting the Pulleniatina record, perhaps related to distance from the equator. Of course, little or nothing can be said about phase shifts between these two records unless a means exists to produce a detailed correlation independent of the signals compared.

Encouraged by the ease with which a good match is achieved between the two records, we next turn to greater detail, attempting peak-to-peak correlation using standardized SAND values (Figs. 17A-17B and 18). The matching was achieved by means of a visual fit of peaks and valleys. A conversion factor for the depth in Hole $805 \mathrm{C}$ was assigned to each sample in Hole $806 \mathrm{~B}$ as the fit was made downcore. From this, the differentials between adjacent samples in Hole $806 \mathrm{~B}$ were calculated. This matching function, the 


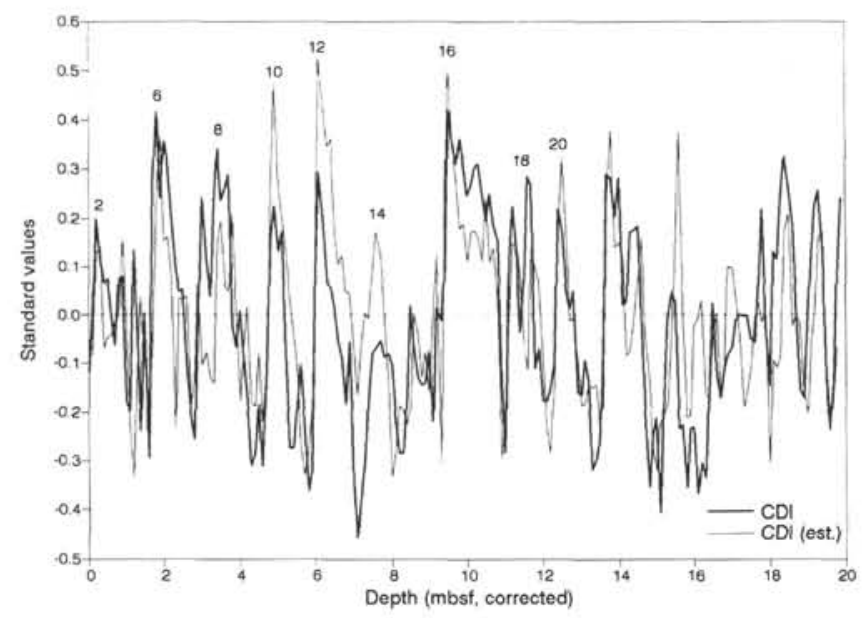

Figure 9. Standardized composite dissolution index (CDI) and standardized $\mathrm{CDI}$ (estimated) vs. corrected depth. $\mathrm{CDI}=(0.5)(\mathrm{CFI})+(0.25)(\% \mathrm{SAND})+$ (0.25) (SAC). CDI (est.) uses $k_{1}=0.55$ and $k_{2}=0.45$ in Equation 4 (described in text). Positive values indicate good preservation.

sequence of instantaneous sedimentation rate ratios (ISRRs), is seen to more or less follow the "crude fit" values proposed earlier but with strong episodic excursions (Fig. 18). Such excursions are produced when matching neighboring peaks in an ad hoc fashion. A Fourier fit to the initial ISRR record removes much of the high-frequency signal and retains the main features of the matching process (Fig. 19). The Fourier fit with 20 harmonics (wavelength $=1 \mathrm{~m}$ ) is retained for comparison with other variables.

The ISRR record that results from matching and smoothing may be compared with the correspondingly filtered $\delta^{18} \mathrm{O}$ record, to check for the effects of glacial-interglacial fluctuations on time scales $>70$ k.y. The comparison (Fig. 20) shows that no clear relationship exists between the variables. For the uppermost $4 \mathrm{~m}$ (ca. $280 \mathrm{ka}$ ), we find that the ISRR between the two sites varies such that Hole 806B has a much greater rate of accumulation during glacials. For earlier periods, on the whole, exactly the reverse is indicated.

An analogous comparison of the ISRR record with the CDI signal from Hole $805 \mathrm{C}$ (Fig. 21) yields somewhat similar observations in terms of phase reversals. The last $250 \mathrm{k} . \mathrm{y}$. are characterized by high sedimentation rate ratios whenever preservation is good. For the 500 k.y. before that, the phase is reversed (down to $11 \mathrm{mbsf}$ ), and a reversed phase is also common below that depth in the hole. However, below 8 mbsf (before $500 \mathrm{ka}$ ), a tendency exists for long-term trends to be in phase, in the sense that good preservation in Hole $805 \mathrm{C}$ means disproportionately high sedimentation rates in Hole $806 \mathrm{~B}$, and vice versa. A priori, one might expect that increased dissolution in Hole $805 \mathrm{C}$ should increase the ISRR between Holes 806 and 805 , if the effects of dissolution do not reach up to shallower depths. Thus, the last $250 \mathrm{k}$.y. would represent the anomalous situation. Unusually high productivity fluctuations (as suggested by the TUM index), combined with unusually strong glacial winnowing activity (as suggested by sand content), may contain the answers to this question.

Based on the 41-k.y. $\delta^{18} \mathrm{O}$ spacing shown in Figure 3, average sedimentation rates per 41-k.y. increment were calculated (Fig. 22). Any partial cycles at the top and bottom of the studied interval were assigned sedimentation rate values based on adjacent complete intervals. The 41-k.y. step-function sedimentation rates were smoothed by constructing a Fourier fit using the first 20 harmonics, and the resulting instantaneous sedimentation rates were used to transform the depth series into a time series (Table 3 ). This analysis of Hole $805 \mathrm{C}$ places the Brunhes/Matuyama boundary near 830,000 ka rather than near 790,000 ka (Shackleton et al., 1990; Izett and Obradovich, 1991; Berger et al., this volume).

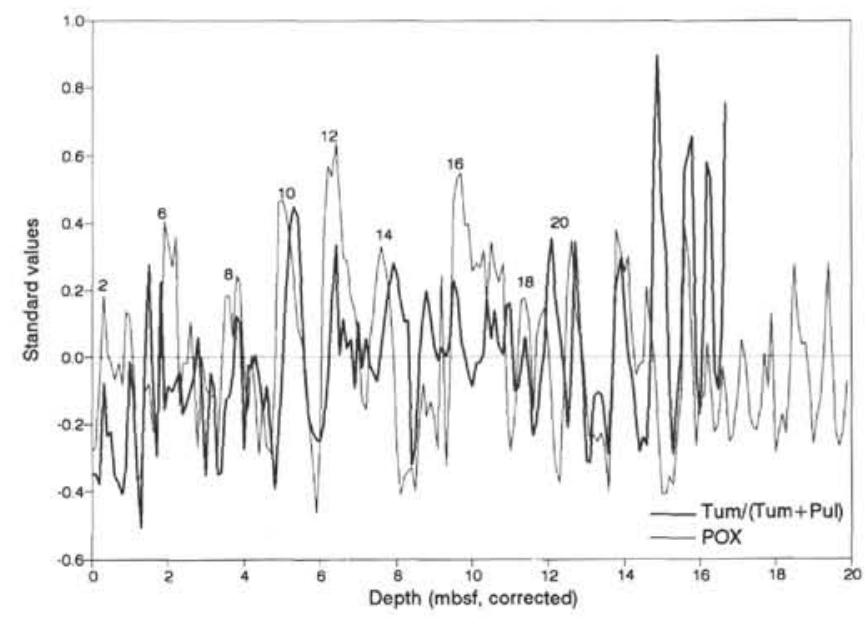

Figure 10. Standardized ratio of number of tests of G. tumida/(Pulleniatina + G. tumida) and standardized $\delta^{18} \mathrm{O}$ Pulleniatina (POX) vs. corrected depth. Positive values indicate higher productivity.

\section{Significance of the Age Model}

The Hole $805 \mathrm{C}$ age model of Berger et al. (this volume) is based on data and procedures identical to ours. Instantaneous sedimentation rates (ISRs) estimated here and by Berger et al. (this volume) are shown in Figure 23 for comparison. Note that Berger et al. show both lower and higher ISRs between 2 and $4 \mathrm{mbsf}$ but much higher ISRs near $11 \mathrm{mbsf}$. The discrepancies between 2 and $4 \mathrm{mbsf}$ cancel for the long term, but those near $11 \mathrm{mbsf}$ do not (Table 3). The discrepancy at $11 \mathrm{mbsf}$ may be explained by the presence of an additional obliquity cycle in our age model, which reduces the ISR in this interval relative to the Berger et al. value. The ISR difference between 2 and 4 mbsf arises despite the fact that data and algorithms are identical. This study treated the entire depth-series as one continuous unit; whereas, Berger et al. treated each core as a separate unit and added synthetic data to the extrapolated ends of each data set. Berger et al. combine the $\delta^{18} \mathrm{O}$ records of Pulleniatina and G. sacculifer for generation of the obliquity-related cycles; we use only the Pulleniatina record, however. Also, the age model used here avoids the discrepancies in age assignments that arise when comparing counts from obliquity and eccentricity cycles at 0.9 to $1 \mathrm{Ma}$, as noted by Berger et al. (this volume).

A test for the two age models and the resulting ISRs in this work might be derived from dissolution patterns. Higher ISRs should be associated with better preservation if preservation is important to net sedimentation rate. However, comparison of our ISRs with CDI (Fig. 24) shows that our ISR model does not consistently respond to observed CDI, making such a test inconclusive.

\section{CONCLUSIONS}

Our results confirm previous observations associating periods of enhanced preservation with glacial periods and with glacial-to-interglacial transitions. In particular, our results show that the record of dissolution is best reconstructed when the state and rate of change of the system based on $\delta^{18} \mathrm{O}$ Pulleniatina are considered. Accounting for the effects of productivity do not improve the estimate of the intensity of dissolution, suggesting that whatever contribution productivity makes to the variability of preservation, it is already contained in the fluctuations of the $\delta^{18} \mathrm{O}$ values of Pulleniatina. Measurements of our productivity indexes suggest that productivity plays a subordinate role in foraminiferal carbonate dissolution, leaving bottom-water saturation as the chief controlling factor. However, a straightforward correlation between the record of preservation and the sedimentation 


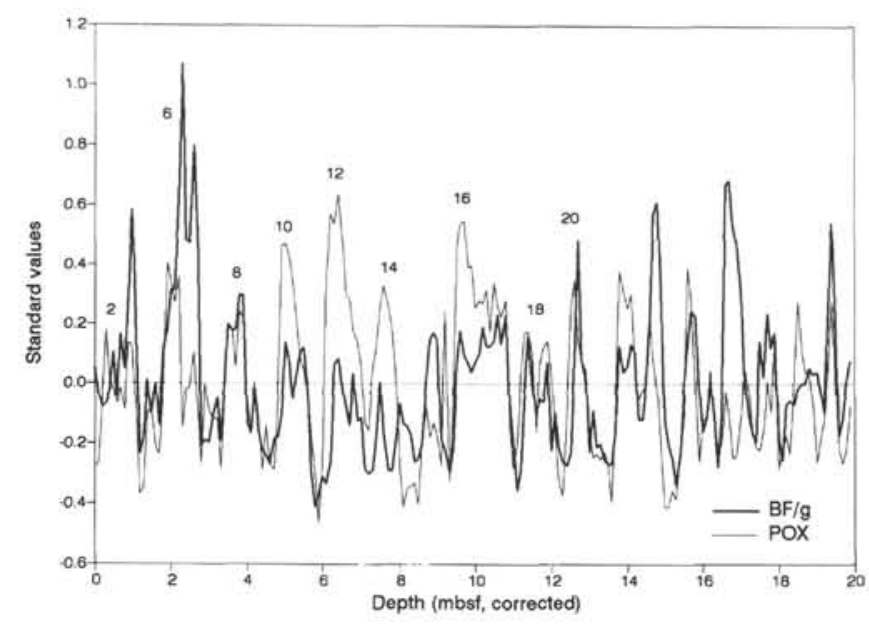

Figure 11. Standardized total number of benthic foraminifers per gram of dry sediment $(\mathrm{BF} / \mathrm{g})$ and standardized $\delta^{18} \mathrm{O}$ Pulleniatina $(\mathrm{POX})$ vs. corrected depth. Positive values indicate higher productivity.

rate stratigraphy of these carbonate-rich sediments could not be established. Also, notable differences are present between the responses of individual dissolution indexes pointing to the variable importance of other processes, which include (but are not limited to) winnowing, bioturbation, and productivity.

\section{ACKNOWLEDGMENTS}

We thank G. Wefer and T. Bickert, University of Bremen, for permitting use of unpublished isotope data. We also thank the anonymous reviewer for critical and constructive reading of the manuscript and Huy Tran for careful sample preparation. This work was supported through National Science Foundation Grant No. OCE 9017717 and U.S. Science Support Program Purchase Order No. 20461.

\section{REFERENCES}

Adelseck, C.G., Jr., 1977. Recent and late Pleistocene sediments from the eastern equatorial Pacific Ocean: sedimentation and dissolution [Ph.D. dissert.]. Univ. of California, San Diego.

Adelseck, C.G., Jr., and Anderson, T.F., 1978. The late Pleistocene record of productivity fluctuations in the eastern equatorial Pacific Ocean. Geology, 6:388-391.

Archer, D., 1991. Modeling the calcite lysocline. J. Geophys. Res., 96:17,03717,050 .

Arrhenius, G., 1952. Properties of the sediment and their distribution: sediment cores from the East Pacific. Rep. Swed. Deep-Sea Exped. 1947-1948, $5: 12-23$.

1 , 1988. Rate of production, dissolution and accumulation of biogenic solids in the ocean. Palaeogeogr., Palaeoclimatol., Palaeoecol., 67:119-146.

Berger, W.H., 1970a. Planktonic foraminifera: selective solution and the lysocline. Mar. Geol., 8:111-138.

, 1970b. Biogenous deep-sea sediments: fractionation by deep-sea circulation. Geol. Soc. Am. Bull., 81:1385-1402.

Foraminiferal Res-sea carbonates: Pleistocene dissolution cycles. J. Foraminiferal Res., 3:187-195.

, 1977. Carbon dioxide excursions and the deep sea record: aspects of the problem. In Andersen, N.R., and Malahoff, A. (Eds.), The Fate of Fossil Fuel $\mathrm{CO}_{2}$ in the Oceans: New York (Plenum), 505-542.

, 1992. Pacific carbonate cycles revisited: arguments for and against productivity control. In Ishizaki, K., and Saito, T. (Eds.), Centenary of Japanese Micropaleontology: Tokyo (Terra Sci.), 15-25.

Berger, W.H., and Keir, R.S., 1984. Glacial-Holocene changes in atmospheric $\mathrm{CO}_{2}$ and the deep-sea record. In Hansen, J.E., and Takahashi, T. (Eds.), Climate Processes and Climate Sensitivity. Am. Geophys. Union, Maurice Ewing Ser., 5:337-351.
Berger, W.H., Kroenke, L.W., Mayer, L.A., and Shipboard Scientific Party, 1991. Ontong Java Plateau, Leg 130: synopsis of major drilling results. In Kroenke, L.W., Berger, W.H., Janecek, T.R., et al., Proc. ODP, Init. Repts., 130: College Station, TX (Ocean Drilling Program), 497-537.

Berger, W.H., and Vincent, E., 1981. Chemostratigraphy and biostratigraphic correlation: exercises in systemic stratigraphy. Oceanol. Acta, Suppl., $4: 115-127$.

Berger, W.H., and Winterer, E.L., 1974. Plate stratigraphy and the fluctuating carbonate line. In Hsü, K.J., and Jenkyns, H.C. (Eds.), Pelagic Sediments on Land and Under the Sea. Spec. Publ. Int. Assoc. Sedimentol., 1:11-48.

Broecker, W.S., and van Donk, J., 1970. Insolation changes, ice volumes and the ${ }^{18} \mathrm{O}$ record in deep-sea sediments. Rev. Geophys. Space Phys., 8:169-198.

Burke, S.K., Berger, W.H., Coulbourn, W.T., and Vincent, E., in press. Benthic foraminifera in Box Core ERDC 112, Ontong Java Plateau. J. Foraminiferal Res.

Chuey, J.M., Rea, D.K., and Pisias, N.G., 1987. Late Pleistocene paleoclimatology of the central equatorial Pacific: a quantitative record of eolian and carbonate deposition. Quat. Res., 28:323-339.

Crowley, T.J., 1985. Late Quaternary changes in the North Atlantic and Atlantic/Pacific comparison. In Sundquist, E.T., and Broecker, W.S. (Eds.), The Carbon Cycle and the Atmospheric $\mathrm{CO}_{2}$ : Natural Variations Archean to Present. Am. Geophys. Union Monogr., 32:271-284.

Emerson, S., and Bender, M., 1981. Carbon fluxes at the sediment-water interface of the deep-sea: calcium carbonate preservation. J. Mar. Res., 39:139-162.

Farrell, J.W., and Prell, W.L., 1989. Climatic change and $\mathrm{CaCO}_{3}$ preservation: an 800,000 year bathymetric reconstruction from the central equatorial Pacific Ocean. Paleoceanography, 4:447-466.

- 1991. Pacific $\mathrm{CaCO}_{3}$ preservation and $\delta^{18} \mathrm{O}$ since 4 Ma: paleoceanic and paleoclimatic implications. Paleoceanography, 6:485-498.

Gardner, J.V., 1982. High-resolution carbonate and organic-carbon stratigraphies for the late Neogene and Quaternary from the western Caribbean and eastern equatorial Pacific. In Prell, W.L., Gardner, J.V., et al., Init. Repts. DSDP, 68: Washington (U.S. Govt. Printing Office), 347-364.

Grötsch, J., Wu, G., and Berger, W.H., 1991. Carbonate cycles in the Pacific: reconstruction of saturation fluctuations. In Einsele, G., Ricken, W., and Seilacher, A. (Eds.), Cycles and Events in Stratigraphy: Heidelberg (Springer-Verlag), 110-125.

Hays, J.D., Saito, T., Opdyke, N.D., and Burckle, L.H., 1969. Pliocene-Pleistocene sediments of the equatorial Pacific: their paleomagnetic, biostratigraphic, and climate record. Geol. Soc. Am. Bull., 8:1481-1514.

Hebbeln, D., Wefer, G., and Berger, W.H., 1990. Pleistocene dissolution fluctuations from apparent depth of deposition in Core ERDC-127P, westequatorial Pacific. Mar. Geol., 165-176.

Herguera, J.C., and Berger, W.H., 1991. Productivity from benthic foraminifera abundance: glacial to postglacial change in the west-equatorial Pacific. Geology, 19:1173-1176.

Imbrie, J., Hays, J.D., Martinson, D.G., McIntyre, A., Mix, A.C., Morley, J.J., Pisias, N.J., Prell, W.L., and Shackleton, N.J., 1984. The orbital theory of Pleistocene climate: support from a revised chronology of the marine $\delta^{18} \mathrm{O}$ record. In Berger, A.L., Imbrie, J., Hays, J., Kukla, G., and Saltzman, B. (Eds.), Milankovitch and Climate (Pt. 2): Dordrecht (D. Reidel), 269-305.

Izett, G.A., and Obradovich, J.D., 1991. Dating of the Matuyama-Brunhes boundary based on ${ }^{40} \mathrm{Ar}^{-39} \mathrm{Ar}$ ages of the Bishop Tuff and Cerro San Luis rhyolite. Geol. Soc. Am., Abstr. Progr., A106.

Johnson, T.C., Hamilton, E.L., and Berger, W.H., 1977. Physical properties of calcareous ooze: control by dissolution at depth. Mar. Geol., 24:259-277.

Keir, R.S., and Berger, W.H., 1983. Atmospheric $\mathrm{CO}_{2}$ content in the last 120,000 years: the phosphate extraction model. J. Geophys. Res., 88:6027-6038.

Le, J., and Shackleton, N.J., 1992. Carbonate dissolution fluctuations in the western equatorial Pacific during the late Quaternary. Paleoceanography, $7: 21-42$.

Luz, B., and Shackleton, N.J., 1975. $\mathrm{CaCO}_{3}$ solution in the tropical east Pacific during the past 130,000 years. In Sliter, W.V., Bé, A.W.H., and Berger, W.H. (Eds.), Dissolution of Deep-Sea Carbonates. Spec. Publ. Cushman Found. Foraminiferal Res., 13:142-150.

Lyle, M., Murray, D.W., Finney, B.P., Dymond, J., Robbins, J.M., and Brooksforce, K., 1988. The record of late Pleistocene biogenic sedimentation in the eastern tropical Pacific Ocean. Paleoceanography, 3:39-59.

Milliman, J.D., 1974. Marine Carbonates: New York (Springer-Verlag).

Moore, T.C., Pisias, N.G., and Dunn, D.A., 1982. Carbonate time series of the Quaternary and late Miocene sediments in the Pacific Ocean: a spectral comparison. Mar. Geol., 46:217-234. 
Moore, T.C., Pisias, N.G., and Heath, G.R., 1977. Climate changes and lags in Pacific carbonate preservation, surface temperature and global ice volume. In Anderson, N.R., and Malahoff, A. (Eds.), The Fate of Fossil Fuel $\mathrm{CO}_{2}$ in the Oceans: New York (Plenum), 145-165.

Olausson, E., 1965. Evidence of climatic changes in North Atlantic deep-sea cores, with remarks on isotopic paleotemperature analysis. Prog. Oceanogr., 3:221-252.

Parker, F.L., and Berger, W.H., 1971. Faunal and solution patterns of planktonic Foraminifera in surface sediments of the South Pacific. Deep-Sea Res., Pt. A, 18:73-107.

Peterson, L.C., and Prell, W.L., 1985a. Carbonate preservation and rates of climatic change: an $800 \mathrm{kyr}$ record from the Indian Ocean. In Sundquist, E.T., and Broecker, W.S. (Eds.), The Carbon Cycle and Atmospheric $\mathrm{CO}_{2}$ : Natural Variations Archean to Present. Am. Geophys. Union Monogr., 32:251-270.

, 1985b. Carbonate dissolution in recent sediments of the eastern equatorial Indian Ocean: preservation patterns and carbonate loss above the lysocline. Mar. Geol., 64:259-290.

Pisias, N.G., 1976. Late Quaternary sediment of the Panama Basin: sedimentation rates, periodicities, and controls of carbonate and opal accumulation. Mem.-Geol. Soc. Am., 145:375-391.

Price, B.A., 1988. Equatorial Pacific sediments: a chemical approach to ocean history [Ph.D. dissert.]. Univ. of California, San Diego.

Schiffelbein, P., 1984. Stable isotope systematics in Pleistocene deep-sea sediment records [Ph.D dissert.]. Univ. of California, San Diego.

Schiffelbein, P., and Dorman, L., 1986. Spectral effects of time-depth nonlinearities in deep sea sediments records: a demodulation technique for realigning time and depth scales. J. Geophys. Res., 91:3821-3835.

Shackleton, N.J., 1977. Carbon-13 in Uvigerina: tropical rainforest history and the equatorial Pacific carbonate dissolution cycles. In Andersen, N.R., and Malahoff, A. (Eds.), The Fate of Fossil Fuel $\mathrm{CO}_{2}$ in the Oceans: New York (Plenum), 401-427.

Shackleton, N.J., Berger, A., and Peltier, W.R., 1990. An alternative astronomical calibration of the lower Pleistocene timescale based on ODP Site 677. Trans. R. Soc. Edinburgh, Earth Sci., 81:251-261.

Shackleton, N.J., and Opdyke, N.D., 1973. Oxygen isotope and palaeomagnetic stratigraphy of equatorial Pacific Core V28-238: oxygen isotope temperatures and ice volumes on a $10^{5}$ year and $10^{6}$ year scale. Quat. Res., $3: 38-55$.

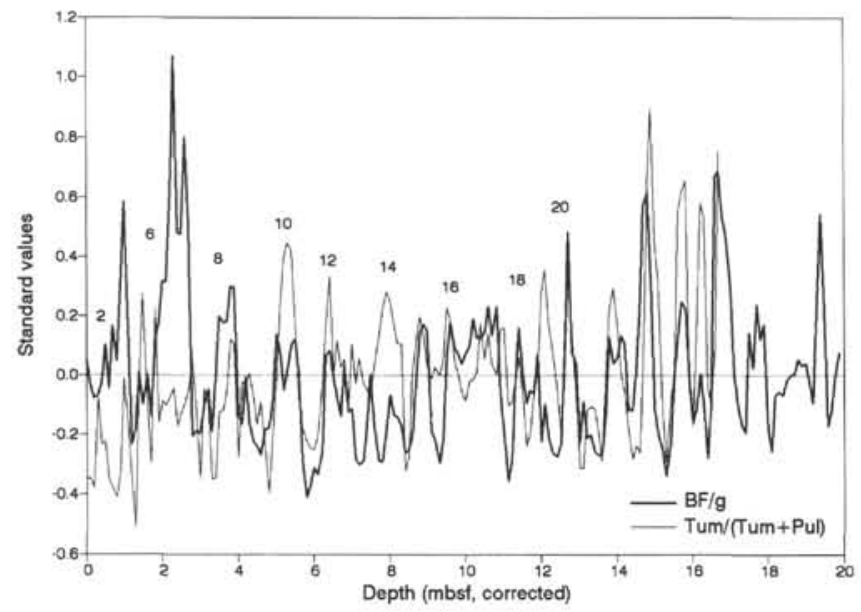

Figure 12. Comparison of productivity indexes: standardized whole tests of G. tumida/(Pulleniatina + G. tumida) and standardized total number of benthic foraminifers per gram of dry sediment (BF/g) vs. corrected depth. Positive values indicate higher productivity.
1976. Oxygen-isotope and paleomagnetic stratigraphy of Pacific core V28-239 Late Pliocene to latest Pleistocene. Mem. - Geol. Soc. Am. 145:449-464.

Shipboard Scientific Party, 1991. Site 805. In Kroenke, L.W., Berger, W.H., Janecek, T.R., et al., Init. Repts. ODP, 130: College Station, TX (Ocean Drilling Program), 223-290.

Thompson, P.R., 1976. Planktonic foraminiferal dissolution and the progress towards a Pleistocene equatorial Pacific transfer function. J. Foraminiferal Res., 6:208-227.

Thompson, P.R., and Saito, T., 1974. Pacific Pleistocene sediments: planktonic foraminifera dissolution cycles and geochronology. Geology, 2:333-335.

Thunell, R.C., 1976. Optimum indices of calcium carbonate dissolution in deep-sea sediments. Geology, 4:525-528.

Valencia, M., 1977. Pacific Pleistocene paleoclimatic stratigraphies: a comparative analysis of results. Quat. Res., 8:339-354.

Vincent, E., 1985. Distribution stratigraphique de la teneur en carbonate dans les sédiments néogènes et quaternaires de l'océan Pacifique. Bull. Soc Geol. Fr., 8:915-924.

Volat, J.L., Pastouret, L., and Vergnaud-Grazzini, C., 1980. Dissolution and carbonate fluctuations in Pleistocene deep-sea cores: a review. Mar. Geol., $34: 1-28$.

Wu, G., and Berger, W.H., 1989. Planktonic foraminifera: differential dissolution and the Quaternary stable isotope record in the west equatorial Pacific. Paleoceanography, 4:181-198.

1991. Pleistocene ${ }^{18} \mathrm{O}$ record from Ontong-Java Plateau: effects of winnowing and dissolution. Mar. Geol., 96:193-209.

Wu, G., Herguera, J.C., and Berger, W.H., 1990. Differential dissolution: modification of late Pleistocene oxygen isotope records in the western equatorial Pacific. Paleoceanography, 5:581-594.

Wu, G., Yasuda, M.K., and Berger, W.H., 1991. Late Pleistocene carbonate stratigraphy on Ontong-Java Plateau in the western equatorial Pacific. Mar. Geol., 99:135-150.

Date of initial report: 21 February 1992

Date of acceptance: 7 September 1992

Ms 130B-008

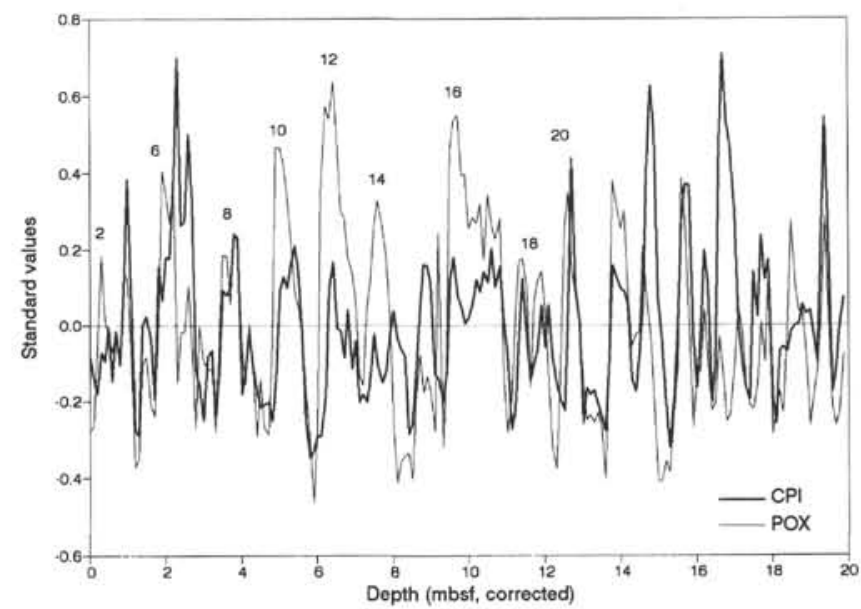

Figure 13. Standardized composite productivity index (CPI) and standardized $\delta^{18} \mathrm{O}$ Pulleniatina (POX) vs. corrected depth. CPI is the standardized mean of the two standardized productivity indexes, G. tumida/(Pulleniatina $+G$. tumida), and total number of benthic foraminifers per gram of dry sediment $(\mathrm{BF} / \mathrm{g})$. Positive CPI values indicate higher productivity. 


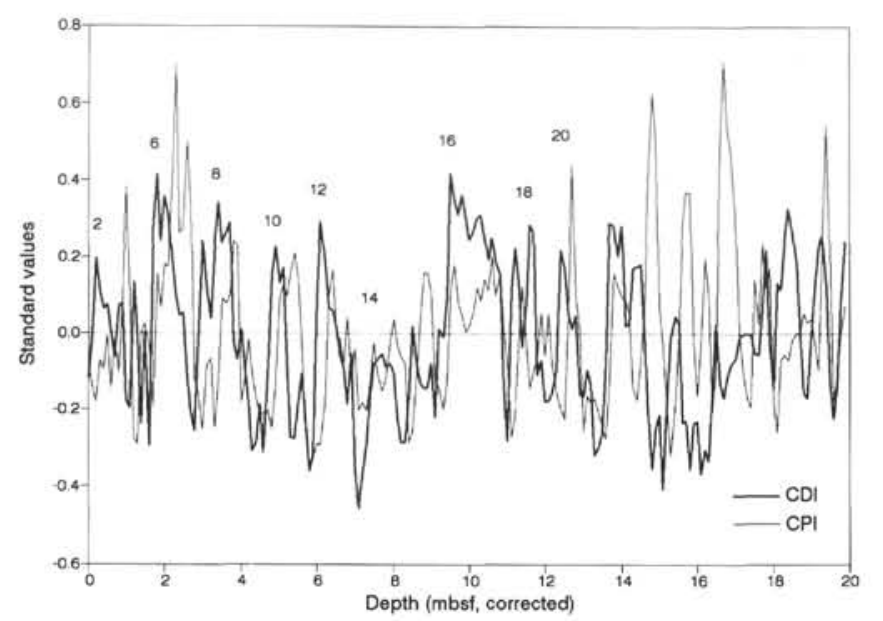

Figure 14. CDI and CPI vs. corrected depth.

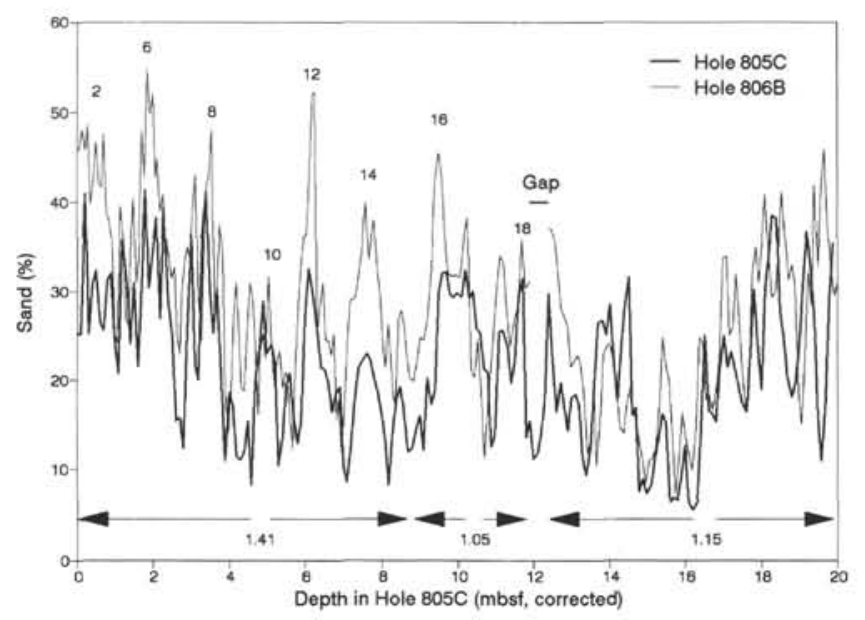

Figure 15. Percent sand at Holes $805 \mathrm{C}$ and $806 \mathrm{~B}$ correlated to corrected depth of Hole $805 \mathrm{C}$ based on linear fit within three separate depth ranges. Values $1.41,1.05$, and 1.15 are sedimentation rate ratios (SRR) applied to depth segments of Hole $805 \mathrm{C}$ for correlation with Hole 806B. Note that a gap must be postulated for Hole 806B to obtain this fit.

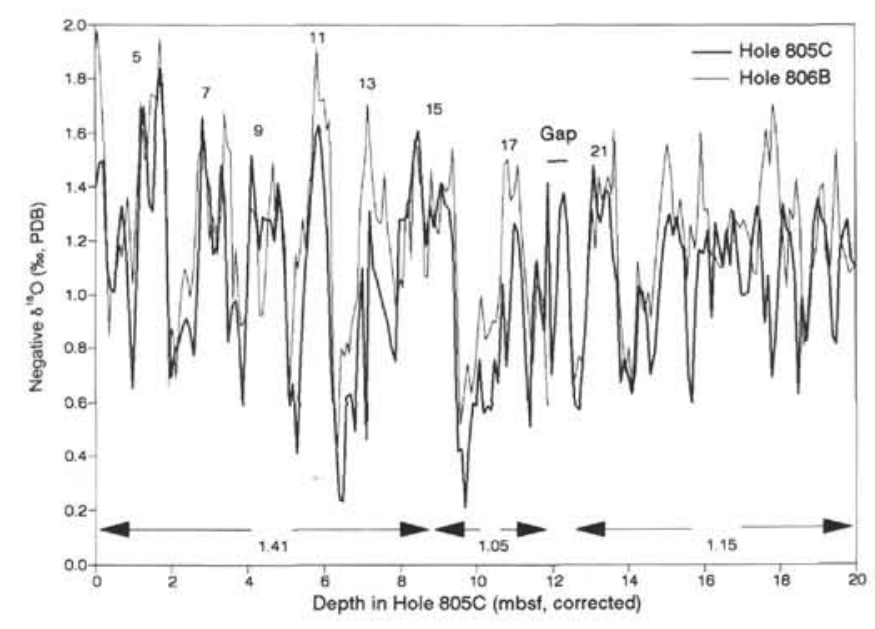

Figure 16. Negative $\delta^{18} \mathrm{O}$ G, sacculifer at Holes $805 \mathrm{C}$ and $806 \mathrm{~B}$ correlated by matching percent sand as shown in Figure 15. 
Table 4. Percent sand ( $>63 \mu \mathrm{m})$ by dry weight, Hole 806B.

\begin{tabular}{|c|c|c|c|c|c|c|c|c|}
\hline $\begin{array}{l}\text { Core, section, } \\
\text { interval }(\mathrm{cm})\end{array}$ & $\begin{array}{l}\text { Depth* } \\
\text { (mbsf) }\end{array}$ & $\begin{array}{l}\text { Sand fraction } \\
>63 \mu \mathrm{m} \\
(\%)\end{array}$ & $\begin{array}{l}\text { Core, section, } \\
\text { interval }(\mathrm{cm})\end{array}$ & $\begin{array}{l}\text { Depth* } \\
\text { (mbsf) }\end{array}$ & $\begin{array}{l}\text { Sand fraction } \\
>63 \mu \mathrm{m} \\
(\%)\end{array}$ & $\begin{array}{l}\text { Core, section, } \\
\text { interval }(\mathrm{cm})\end{array}$ & $\begin{array}{l}\text { Depth* } \\
\text { (mbsf) }\end{array}$ & $\begin{array}{l}\text { Sand fraction } \\
>63 \mu \mathrm{m} \\
(\%)\end{array}$ \\
\hline $1 \mathrm{H}-1,9-11$ & 0.09 & 45.8 & $2 \mathrm{H}-1,59-61$ & 7.09 & 31.9 & $2 \mathrm{H}-5,108-110$ & 13.58 & 42.5 \\
\hline $1 \mathrm{H}-\mathrm{I}, 19-21$ & 0.19 & 48.3 & $2 \mathrm{H}-1,69-71$ & 7.19 & 27.0 & $2 \mathrm{H}-5,119-121$ & 13.69 & 33.5 \\
\hline $1 \mathrm{H}-1,29-31$ & 0.29 & 45.3 & $2 \mathrm{H}-1,79-81$ & 7.29 & 20.6 & $2 \mathrm{H}-5,129-131$ & 13.79 & 31.9 \\
\hline $1 \mathrm{H}-1,39-41$ & 0.39 & 50.1 & $2 \mathrm{H}-1,89-91$ & 7.39 & 19.9 & $2 \mathrm{H}-5,139-141$ & 13.89 & 31.7 \\
\hline $1 \mathrm{H}-1,49-51$ & 0.49 & 40.2 & $2 \mathrm{H}-1,99-101$ & 7.49 & 24.3 & $2 \mathrm{H}-5,148-150$ & 13.98 & 32.0 \\
\hline $1 \mathrm{H}-1,59-61$ & 0.59 & 41,4 & $2 \mathrm{H}-1,109-111$ & 7.59 & 21.1 & $2 \mathrm{H}-6,9-11$ & 14.09 & 31.6 \\
\hline IH-1, 69-71 & 0.69 & 46.8 & $2 \mathrm{H}-1,119-121$ & 7.69 & 18.5 & $2 \mathrm{H}-6,19-21$ & 14.19 & 33.1 \\
\hline $1 \mathrm{H}-1,79-81$ & 0.79 & 44.3 & $2 \mathrm{H}-\mathrm{I}, 129-\mathrm{I} 3 \mathrm{I}$ & 7.79 & 22.3 & $2 \mathrm{H}-6,29-31$ & 14.29 & 42.0 \\
\hline $1 \mathrm{H}-1,89-91$ & 0.89 & 38.5 & $2 \mathrm{H}-1,139-141$ & 7.89 & 19.8 & $2 \mathrm{H}-6,36-38$ & 14.36 & 28.5 \\
\hline $1 \mathrm{H}-1,99-101$ & 0.99 & 48.7 & $2 \mathrm{H}-\mathrm{I}, 148-150$ & 7.98 & 15.8 & $2 \mathrm{H}-6,49-51$ & 14.49 & 19.9 \\
\hline $1 \mathrm{H}-1,109-111$ & 1.09 & 41.8 & $2 \mathrm{H}-2,9-11$ & 8.09 & 12.2 & $2 \mathrm{H}-6,59-61$ & 14.59 & 23.1 \\
\hline $1 \mathrm{H}-1,119-121$ & 1.19 & 36.8 & $2 \mathrm{H}-2,19-21$ & 8.19 & 22.7 & $2 \mathrm{H}-6,69-71$ & 14.69 & 23.8 \\
\hline $1 \mathrm{H}-1,129-131$ & 1.29 & 39.8 & $2 \mathrm{H}-2,29-31$ & 8.29 & 28.7 & $2 \mathrm{H}-6,79-81$ & 14.79 & 13.3 \\
\hline $1 \mathrm{H}-\mathrm{I}, 139-141$ & 1.39 & 30.5 & $2 \mathrm{H}-2,39-41$ & 8.39 & 33.6 & $2 \mathrm{H}-6,89-91$ & 14.89 & 12.5 \\
\hline $1 \mathrm{H}-1,148-150$ & 1.48 & 23.4 & $2 \mathrm{H}-2,49-51$ & 8.49 & 36.3 & $2 \mathrm{H}-6,99-101$ & 14.99 & 21.6 \\
\hline $1 \mathrm{H}-2,9-11$ & 1.59 & 28.2 & $2 \mathrm{H}-2,59-61$ & 8.59 & 35.1 & $2 \mathrm{H}-6,108-110$ & 15.08 & 24.1 \\
\hline $1 \mathrm{H}-2,19-21$ & 1.69 & 45.2 & $2 \mathrm{H}-2,69-71$ & 8.69 & 43.1 & $2 \mathrm{H}-6,119-121$ & 15.19 & 31.4 \\
\hline IH-2, 29-31 & 1.79 & 29.4 & $2 \mathrm{H}-2,79-81$ & 8.79 & 47.1 & $2 \mathrm{H}-6,129-131$ & 15.29 & 34.3 \\
\hline $1 \mathrm{H}-2,39-41$ & 1.89 & 27.1 & $2 \mathrm{H}-2,89-91$ & 8.89 & 59.1 & $2 \mathrm{H}-6,139-141$ & 15.39 & 32.9 \\
\hline IH-2, 49-51 & 1.99 & 26.6 & $2 \mathrm{H}-2,99-101$ & 8.99 & 30.7 & $2 \mathrm{H}-6,148-150$ & 15.48 & 28.2 \\
\hline $1 \mathrm{H}-2,59-61$ & 2.09 & 36.7 & $2 \mathrm{H}-2,109-111$ & 9.09 & 25.5 & $2 \mathrm{H}-7,9-11$ & 15.59 & 23.8 \\
\hline IH-2, 69-71 & 2.19 & 40.4 & $2 \mathrm{H}-2,119-121$ & 9.19 & 33.8 & $2 \mathrm{H}-7,19-2 \mathrm{I}$ & 15.69 & 26.9 \\
\hline $1 \mathrm{H}-2,79-81$ & 2.29 & 29.7 & $2 \mathrm{H}-2,129-131$ & 9.29 & 24.7 & $2 \mathrm{H}-7,27-29$ & 15.77 & 25.9 \\
\hline $1 \mathrm{H}-2,89-91$ & 2.39 & 33.7 & $2 \mathrm{H}-2,139-141$ & 9.39 & 26.1 & $2 \mathrm{H}-7,39-41$ & 15.89 & 36.2 \\
\hline $1 \mathrm{H}-2,99-101$ & 2.49 & 48.0 & $2 \mathrm{H}-2,148-150$ & 9.48 & 23.4 & $2 \mathrm{H}-7,49-5 \mathrm{I}$ & 15.99 & 30.9 \\
\hline $1 \mathrm{H}-2,108-110$ & 2.58 & 41.6 & $2 \mathrm{H}-3,9-11$ & 9.59 & 23.1 & $2 \mathrm{H}-7,59-61$ & 16.09 & 30.4 \\
\hline $1 \mathrm{H}-2,119-121$ & 2.69 & 55.0 & $2 \mathrm{H}-3,19-2 \mathrm{I}$ & 9.69 & 25.5 & $2 \mathrm{H}-7,67-69$ & 16.17 & 33.6 \\
\hline $1 \mathrm{H}-2,129-131$ & 2.79 & 49.2 & $2 \mathrm{H}-3,29-31$ & 9.79 & 12.6 & $3 \mathrm{H}-1,9-11$ & 16.09 & 36.5 \\
\hline $1 \mathrm{H}-2,139-141$ & 2.89 & 53.9 & $2 \mathrm{H}-3,39-41$ & 9.89 & 18.9 & $3 \mathrm{H}-1,19-2 \mathrm{I}$ & 16.19 & 32.4 \\
\hline $1 \mathrm{H}-2,148-150$ & 2.98 & 42.4 & $2 \mathrm{H}-3,49-51$ & 9.99 & 10.9 & $3 \mathrm{H}-1,39-41$ & 16.39 & 26.6 \\
\hline $1 \mathrm{H}-3,9-11$ & 3.09 & 46.4 & $2 \mathrm{H}-3,59-61$ & 10.09 & 17.8 & $3 \mathrm{H}-1,49-51$ & 16.49 & 28.3 \\
\hline $1 \mathrm{H}-3,19-21$ & 3.19 & 38.4 & $2 \mathrm{H}-3,69-71$ & 10.19 & 23.8 & $3 \mathrm{H}-1,59-6 \mathrm{I}$ & 16.59 & 21.3 \\
\hline $1 \mathrm{H}-3,29-31$ & 3.29 & 42.3 & $2 \mathrm{H}-3,79-81$ & 10.29 & 28.9 & $3 \mathrm{H}-1,69-71$ & 16.69 & 23.7 \\
\hline $1 \mathrm{H}-3,39-41$ & 3.39 & 36.5 & $2 \mathrm{H}-3,89-91$ & 10.39 & 29.1 & $3 \mathrm{H}-1,79-81$ & 16.79 & 20.2 \\
\hline $1 \mathrm{H}-3,49-51$ & 3.49 & 36.4 & $2 \mathrm{H}-3,99-101$ & 10.49 & 29.2 & $3 \mathrm{H}-1,89-91$ & 16.89 & 25.1 \\
\hline $1 \mathrm{H}-3,59-61$ & 3.59 & 30.4 & $2 \mathrm{H}-3,109-111$ & 10.59 & 31.1 & $3 \mathrm{H}-1,99-101$ & 16.99 & 19.8 \\
\hline $1 \mathrm{H}-3,69-71$ & 3.69 & 34.2 & $2 \mathrm{H}-3,119-121$ & 10.69 & 33.7 & $3 \mathrm{H}-1,107-109$ & 17.07 & 18.5 \\
\hline $1 \mathrm{H}-3,79-81$ & 3.79 & 28.7 & $2 \mathrm{H}-3,129-131$ & 10.79 & 36.2 & $3 \mathrm{H}-1,118-120$ & 17.18 & 11.2 \\
\hline $1 \mathrm{H}-3,89-91$ & 3.89 & 23.0 & $2 \mathrm{H}-3,139-141$ & 10.89 & 40.0 & $3 \mathrm{H}-1,129-131$ & 17.29 & 13.9 \\
\hline $1 \mathrm{H}-3,99-101$ & 3.99 & 25.0 & $2 \mathrm{H}-3,148-150$ & 10.98 & 38.2 & $3 \mathrm{H}-1,139-141$ & 17.39 & 18.4 \\
\hline $1 \mathrm{H}-3,108-110$ & 4.08 & 32.6 & $2 \mathrm{H}-4,9-11$ & 11.09 & 31.2 & $3 \mathrm{H}-1,148-150$ & 17.48 & 10.4 \\
\hline $1 \mathrm{H}-3,114-116$ & 4.14 & 33.1 & $2 \mathrm{H}-4,19-21$ & 11.19 & 41.9 & $3 \mathrm{H}-2,9-11$ & 17.59 & 15.7 \\
\hline $1 \mathrm{H}-4,9-11$ & 4.59 & 42.8 & $2 \mathrm{H}-4,29-31$ & 11.29 & 32.7 & $3 \mathrm{H}-2,19-2 \mathrm{I}$ & 17.69 & 22.4 \\
\hline $1 \mathrm{H}-4,19-21$ & 4.69 & 26.9 & $2 \mathrm{H}-4,39-41$ & 11.39 & 33.1 & $3 \mathrm{H}-2,39-4 \mathrm{I}$ & 17.89 & 24.7 \\
\hline $\mid \mathrm{H}-4,29-31$ & 4.79 & 25.0 & $2 \mathrm{H}-4,49-51$ & 11.49 & 27.7 & $3 \mathrm{H}-2,52-54$ & 18.02 & 21.7 \\
\hline $1 \mathrm{H}-4,39-41$ & 4.89 & 35.5 & $2 \mathrm{H}-4,59-61$ & 11.59 & 23.0 & $3 \mathrm{H}-2,61-63$ & 18.11 & 21.8 \\
\hline $1 \mathrm{H}-4,49-51$ & 4.99 & 42.3 & $2 \mathrm{H}-4,69-71$ & 11.69 & 21.4 & $3 \mathrm{H}-2,69-71$ & 18.19 & 15.8 \\
\hline $1 \mathrm{H}-4,59-61$ & 5.09 & 42.6 & $2 \mathrm{H}-4,79-81$ & 11.79 & 26.8 & $3 \mathrm{H}-2,79-81$ & 18.29 & 15.2 \\
\hline $1 \mathrm{H}-4,69-71$ & 5.19 & 49.8 & $2 \mathrm{H}-4,89-91$ & 11.89 & 21.7 & $3 \mathrm{H}-2,88-90$ & 18.38 & 13.9 \\
\hline $1 \mathrm{H}-4,79-81$ & 5.29 & 28.1 & $2 \mathrm{H}-4,99-101$ & 11.99 & 18.4 & $3 \mathrm{H}-2,99-101$ & 18.49 & 18.6 \\
\hline $1 \mathrm{H}-4,89-91$ & 5.39 & 29.5 & $2 \mathrm{H}-4,108-110$ & 12.08 & 15.6 & $3 \mathrm{H}-2,109-111$ & 18.59 & 19.1 \\
\hline $1 \mathrm{H}-4,99-101$ & 5.49 & 36.6 & $2 \mathrm{H}-4,119-121$ & 12.19 & 26.5 & $3 \mathrm{H}-2,118-120$ & 18.68 & 16.8 \\
\hline $1 \mathrm{H}-4,108-110$ & 5.58 & 37.5 & $2 \mathrm{H}-4,129-131$ & 12.29 & 27.9 & $3 \mathrm{H}-2,129-131$ & 18.79 & 14.9 \\
\hline $1 \mathrm{H}-4,118-120$ & 5.68 & 23.4 & $2 \mathrm{H}-4,139-141$ & 12.39 & 27.1 & $3 \mathrm{H}-3,9-11$ & 19.09 & 8.2 \\
\hline $1 \mathrm{H}-4,129-131$ & 5.79 & 14.4 & $2 \mathrm{H}-5,9-11$ & 12.59 & 20.3 & $3 \mathrm{H}-3,19-21$ & 19.19 & 10.8 \\
\hline $1 \mathrm{H}-4,139-141$ & 5.89 & 16.3 & $2 \mathrm{H}-5,19-21$ & 12.69 & 20.8 & $3 \mathrm{H}-3,39-41$ & 19.39 & 11.5 \\
\hline $1 \mathrm{H}-4,148-150$ & 5.98 & 23.4 & $2 \mathrm{H}-5,29-31$ & 12.79 & 19.3 & $3 \mathrm{H}-3,49-51$ & 19.49 & 15.3 \\
\hline $1 \mathrm{H}-5,9-11$ & 6.09 & 30.3 & $2 \mathrm{H}-5,39-41$ & 12.89 & 25.9 & $3 \mathrm{H}-3,59-61$ & 19.59 & 22.1 \\
\hline IH-5, 19-2। & 6.19 & 29.4 & $2 \mathrm{H}-5,49-51$ & 12.99 & 22.7 & $3 \mathrm{H}-3,69-71$ & 19.69 & 25.3 \\
\hline $1 \mathrm{H}-5,29-31$ & 6.29 & 21.8 & $2 \mathrm{H}-5,59-61$ & 13.09 & 26.6 & $3 \mathrm{H}-3,79-81$ & 19.79 & 18.7 \\
\hline $2 \mathrm{H}-1,9-11$ & 6.59 & 25.0 & $2 \mathrm{H}-5,69-71$ & 13.19 & 26.9 & $3 \mathrm{H}-3,89-91$ & 19.89 & 20.9 \\
\hline $2 \mathrm{H}-1,19-21$ & 6.69 & 15.9 & $2 \mathrm{H}-5,79-81$ & 13.29 & 36.2 & $3 \mathrm{H}-3,99-101$ & 19.99 & 10.8 \\
\hline $2 \mathrm{H}-1,29-31$ & 6.79 & 21.8 & $2 \mathrm{H}-5,89-91$ & 13.39 & 43.8 & $3 \mathrm{H}-3,107-109$ & 20.07 & 7.2 \\
\hline $2 \mathrm{H}-1,39-41$ & 6.89 & 26.7 & $2 \mathrm{H}-5,99-10 \mathrm{I}$ & 13.49 & 45.3 & $3 \mathrm{H}-3,118-120$ & 20.18 & 11.4 \\
\hline $2 \mathrm{H}-1,49-51$ & 6.99 & 19.7 & & & & & & \\
\hline
\end{tabular}

*ODP depth assignment $=$ depth has not been adjusted. 

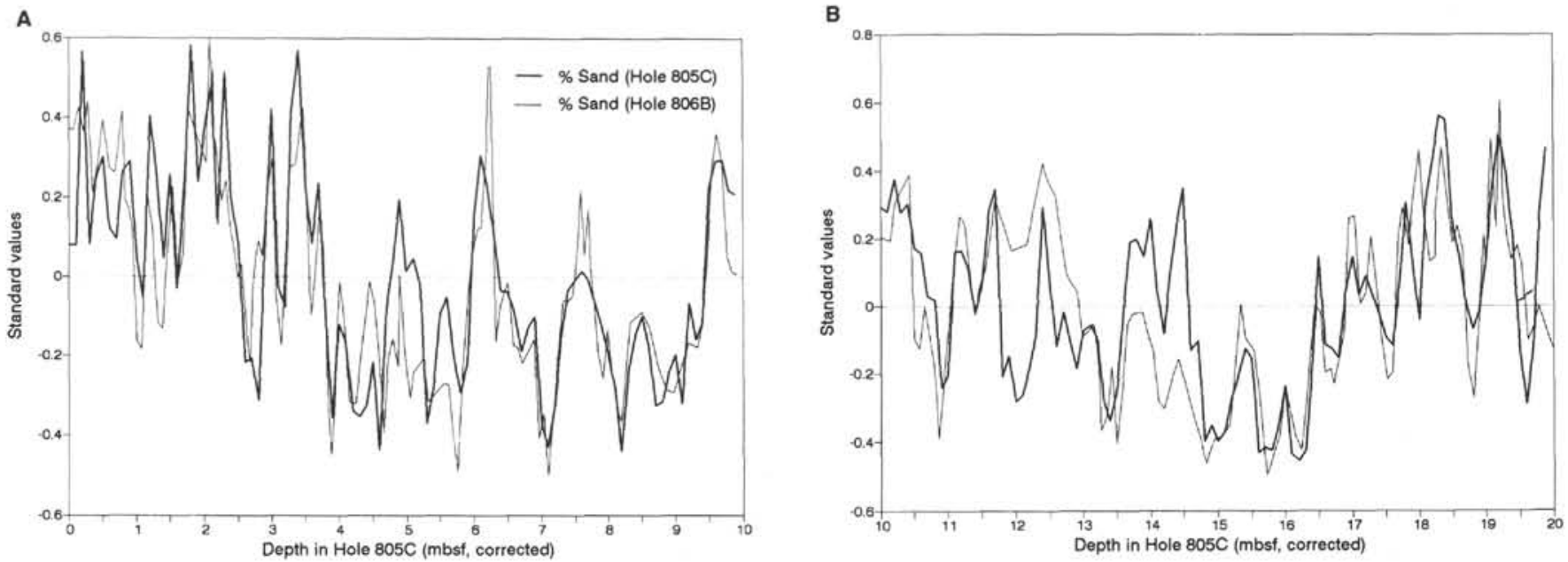

Figure 17. A, B. Standardized percent sand, Holes 805C and 806B, with Hole 806B data correlated to corrected depth of Hole 805C using peak-to-peak fit. Figures $17 \mathrm{~A}$ and $17 \mathrm{~B}$ show data for $0-10$ and $10-20 \mathrm{mbsf}$ (corrected), respectively.

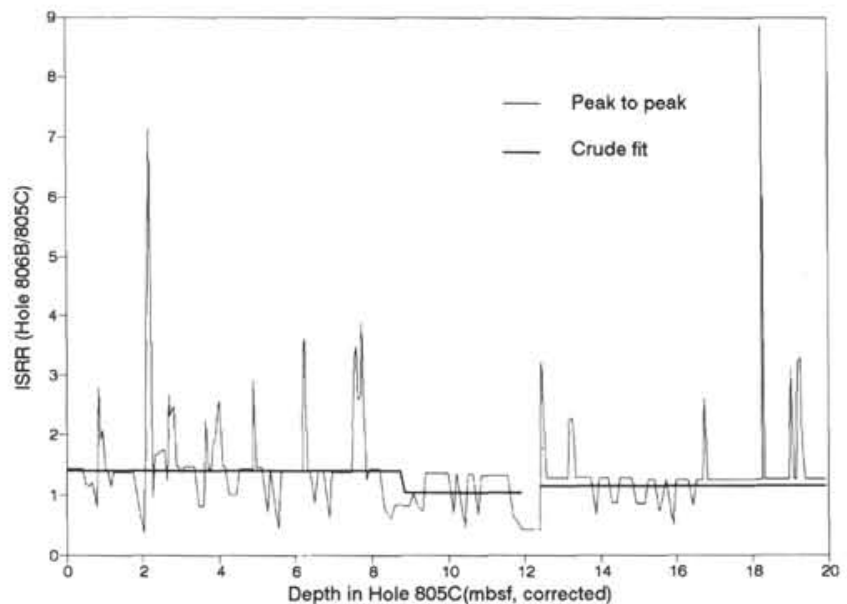

Figure 18. Instantaneous sedimentation rate ratios (ISRR), Holes 806B/805C, based on peak-to-peak fit of percent sand records (Figs. 17A-17B) and on crude fit shown in Figure 15 vs. corrected depth for Hole 805C.

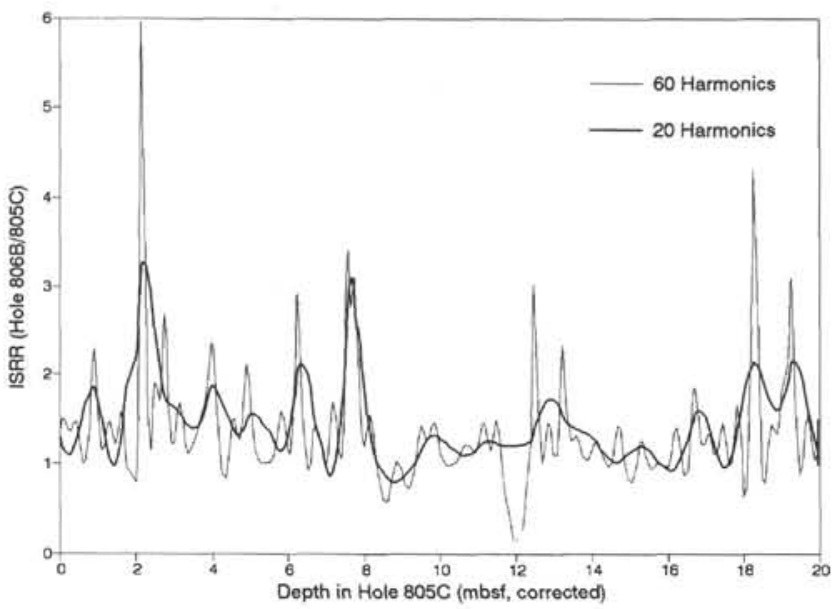

Figure 19. Fourier reconstruction of ISRR Holes $806 \mathrm{~B} / 805 \mathrm{C}$, based on peakto-peak fit (Fig. 18) using the first 60 and 20 harmonics vs. corrected depth for Hole $805 \mathrm{C}$.

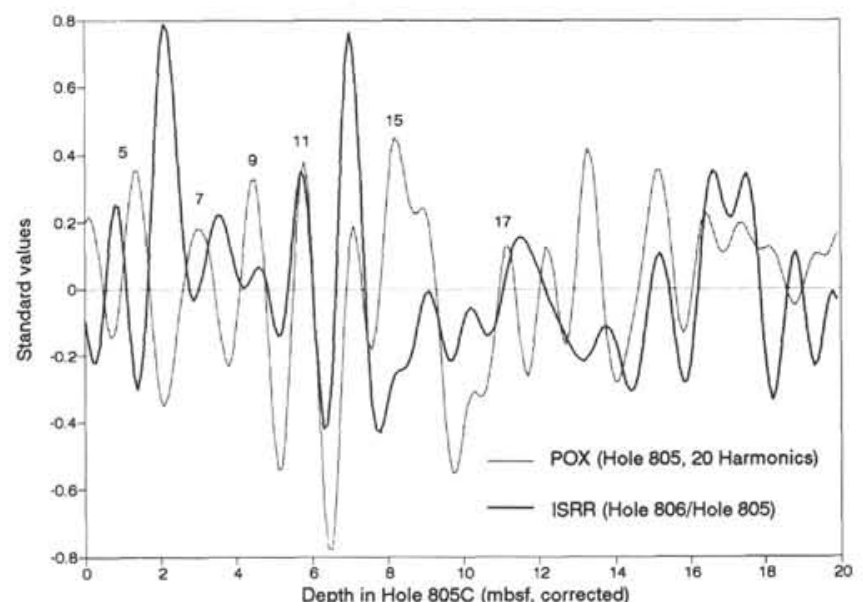

Figure 20. Fourier reconstruction of ISRR Holes $806 \mathrm{~B} / 805 \mathrm{C}$ (Fig. 19) and $\delta^{18} \mathrm{O}$ Pulleniatina (both using the first 20 harmonics) vs. corrected depth, Hole $805 \mathrm{C}$.

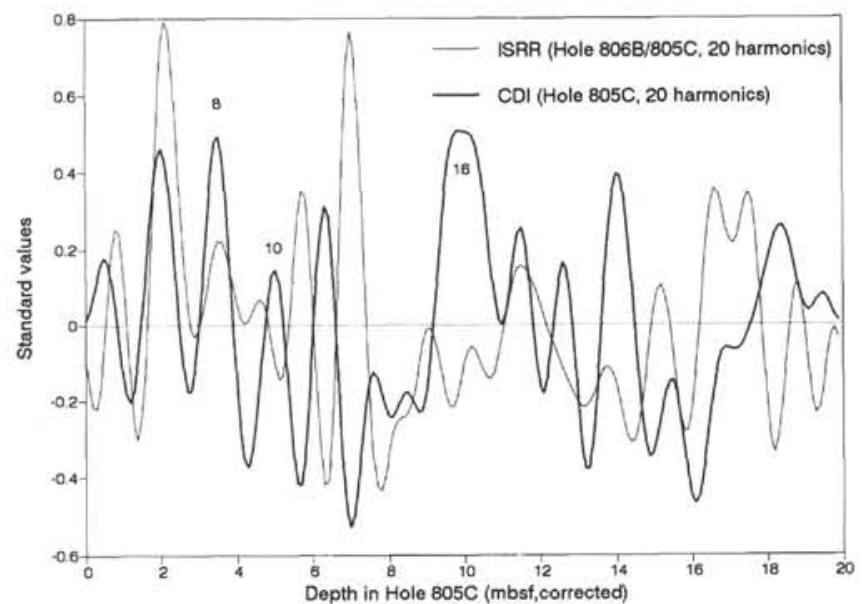

Figure 21. Fourier reconstructions of ISRR Holes 806B/805C (Fig. 19) and CDI (both using the first 20 harmonics) vs. corrected depth for Hole $805 \mathrm{C}$. Positive CDI values indicate good preservation. 


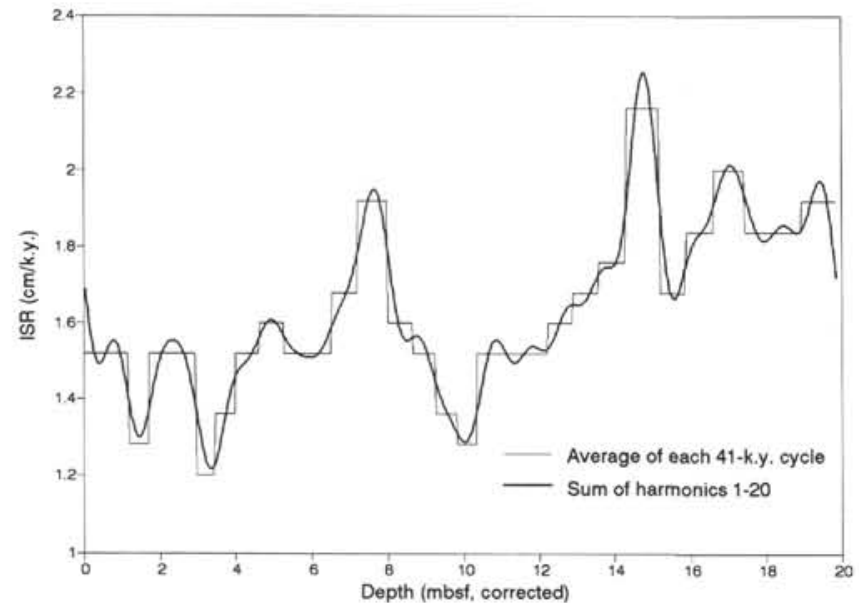

Figure 22. Instantaneous sedimentation rates (ISR) for Hole $805 \mathrm{C}$ based on counting 41-k.y. cycles (Fig. 3). The figure shows both the average sedimentation rate per 41-k.y. cycle and the Fourier fit of this curve using the first 20 harmonics vs. corrected depth. The association of age with depth based on these instantaneous sedimentation rates is shown in Table 3.

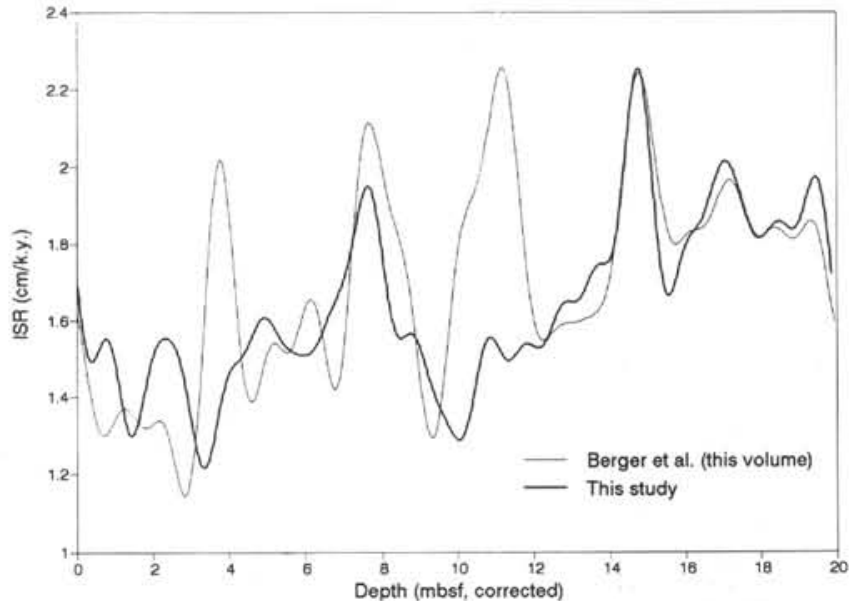

Figure 23. Comparison of instantaneous sedimentation rates (ISR) for Hole $805 \mathrm{C}$ resulting from Berger et al. (this volume, using $\delta^{18} \mathrm{O}$ G. sacculifer) with those of this paper (using $\delta^{18} \mathrm{O}$ Pulleniatina) vs. corrected depth.

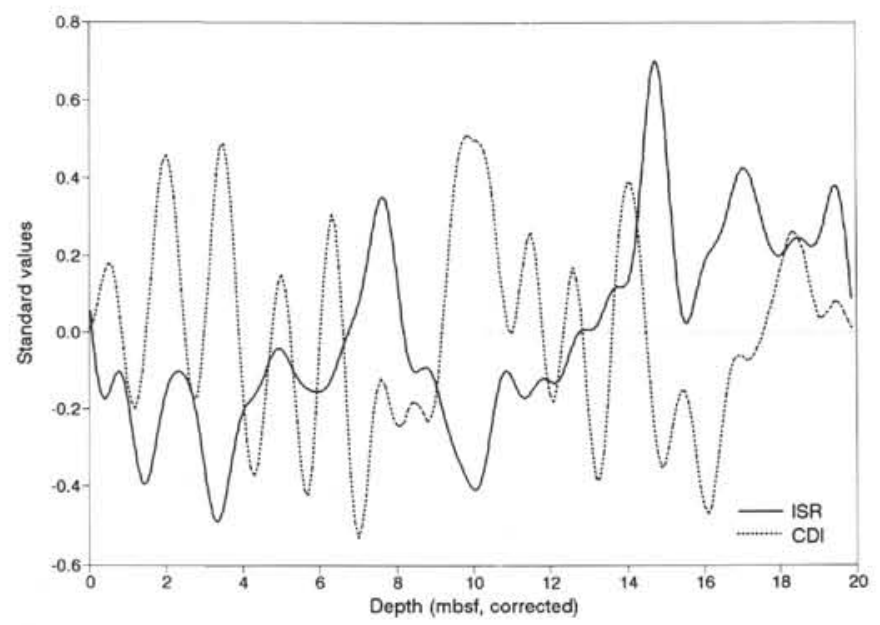

Figure 24. Comparison of instantaneous sedimentation rates (ISR) with CDI for Hole 805C. ISR and CDI plotted vs. corrected depth for Hole 805C. 
APPENDIX

Planktonic Foraminiferal Abundances, Hole 805C

\begin{tabular}{|c|c|c|c|c|c|c|c|c|c|c|c|}
\hline $\begin{array}{l}\text { Core, section, } \\
\text { interval }(\mathrm{cm})\end{array}$ & $\begin{array}{l}\text { Depth* } \\
\text { (mbsf) }\end{array}$ & $\begin{array}{c}\text { Globigerinoides } \\
\text { sacculifer } \\
(\# / g m)\end{array}$ & $\begin{array}{l}\text { Pulleniatina } \\
\text { ("\#/gm) }\end{array}$ & $\begin{array}{c}\text { Globorotalia } \\
\text { menardii } \\
(\# / g m)\end{array}$ & $\begin{array}{c}\text { Globorotalia } \\
\text { tumida } \\
(\# / g m)\end{array}$ & $\begin{array}{l}\text { Core, section, } \\
\text { interval }(\mathrm{cm})\end{array}$ & $\begin{array}{l}\text { Depth* } \\
\text { (mbsf) }\end{array}$ & $\begin{array}{c}\text { Globigerinoides } \\
\text { sacculifer } \\
\text { (\#/gm) }\end{array}$ & $\begin{array}{l}\text { Pulleniatina } \\
(\# / g m)\end{array}$ & $\begin{array}{c}\text { Globorotalia } \\
\text { menardii } \\
(\# / g m)\end{array}$ & $\begin{array}{c}\text { Globorotalia } \\
\text { numida } \\
(\# / g m)\end{array}$ \\
\hline $1 \mathrm{H}-1,13-15$ & 0.13 & 356 & 772 & 71 & 91 & $2 \mathrm{H}-1,139-141$ & 9.19 & 30 & 120 & 36 & 38 \\
\hline $1 \mathrm{H}-1,21-23$ & 0.21 & 251 & 244 & 25 & 25 & $2 \mathrm{H}-1,139-141$ & 9.19 & 29 & 121 & 37 & 36 \\
\hline $1 \mathrm{H}-1,29-31$ & 0.29 & 104 & 121 & 17 & 35 & $2 \mathrm{H}-1,148-150$ & 9.28 & 98 & 176 & 60 & 61 \\
\hline 1H-1, 39-41 & 0.39 & 87 & 179 & 15 & 34 & $2 \mathrm{H}-2,9-11$ & 9.39 & 101 & 188 & 61 & 62 \\
\hline $1 \mathrm{H}-1,49-51$ & 0.49 & 142 & 272 & 40 & 55 & $2 \mathrm{H}-2,19-21$ & 9.49 & 66 & 151 & 65 & 50 \\
\hline $1 \mathrm{H}-1,59-61$ & 0.59 & 88 & 302 & 22 & 42 & $2 \mathrm{H}-2,29-31$ & 9.59 & 271 & 141 & 93 & 70 \\
\hline $1 \mathrm{H}-1,69-71$ & 0.69 & 78 & 296 & 48 & 32 & $2 \mathrm{H}-2,39-41$ & 9.69 & 268 & 162 & 101 & 86 \\
\hline $1 \mathrm{H}-1,69-71$ & 0.69 & 71 & 301 & 48 & 33 & $2 \mathrm{H}-2,49-51$ & 9.79 & 270 & 232 & 135 & 90 \\
\hline $1 \mathrm{H}-1,89-91$ & 0.89 & 164 & 314 & 26 & 29 & $2 \mathrm{H}-2,59-61$ & 9.89 & 222 & 216 & 131 & 79 \\
\hline $1 \mathrm{H}-1,89-91$ & 0.89 & 166 & 326 & 23 & 33 & $2 \mathrm{H}-2,69-71$ & 9.99 & 255 & 205 & 139 & 65 \\
\hline $1 \mathrm{H}-1,89-91$ & 0.89 & 168 & 322 & 31 & 24 & $2 \mathrm{H}-2,79-81$ & 10.09 & 242 & 255 & 65 & 75 \\
\hline $1 \mathrm{H}-1,99-101$ & 0.99 & 85 & 211 & 44 & 69 & $2 \mathrm{H}-2,89-91$ & 10.19 & 192 & 291 & 73 & 78 \\
\hline $1 \mathrm{H}-1,109-111$ & 1.09 & 15 & 182 & 37 & 39 & $2 \mathrm{H}-2,99-101$ & 10.29 & 198 & 253 & 63 & 83 \\
\hline $1 \mathrm{H}-1,119-121$ & 1.19 & 120 & 184 & 29 & 47 & $2 \mathrm{H}-2,109-111$ & 10.39 & 177 & 256 & 101 & 82 \\
\hline $1 \mathrm{H}-1,129-131$ & 1.29 & 383 & 346 & 8 & 11 & $2 \mathrm{H}-2,119-121$ & 10.49 & 129 & 203 & 83 & 70 \\
\hline 1H-1, 143-145 & 1.43 & 26 & 161 & 22 & 35 & $2 \mathrm{H}-2,129-131$ & 10.59 & 152 & 204 & 80 & 95 \\
\hline IH-2,4-6 & 1.54 & 127 & 129 & 29 & 72 & $2 \mathrm{H}-2,139-141$ & 10.69 & 134 & 187 & 62 & 70 \\
\hline $\mid \mathrm{H}-2,19-21$ & 1.69 & II & 154 & 26 & 30 & $2 \mathrm{H}-2,148-150$ & 10.78 & 177 & 218 & 103 & 95 \\
\hline $1 \mathrm{H}-2,29-31$ & 1.79 & 269 & 168 & 94 & 27 & $2 \mathrm{H}-3,9-11$ & 10.89 & 102 & 157 & 73 & 60 \\
\hline IH-2, 39-41 & 1.89 & 341 & 220 & 124 & 130 & $2 \mathrm{H}-3,19-2 \mathrm{I}$ & 10.99 & 140 & 212 & 86 & 56 \\
\hline IH-2, 49-51 & 1.99 & 169 & 143 & 87 & 32 & $2 \mathrm{H}-3,29-3 \mathrm{I}$ & 11.09 & 56 & 117 & 58 & 55 \\
\hline IH-2, 49-51 & 1.99 & 173 & 140 & 75 & 42 & $2 \mathrm{H}-3,29-31$ & 11.09 & 59 & 121 & 64 & 49 \\
\hline IH-2, 59-61 & 2.09 & 425 & 190 & 66 & 52 & $2 \mathrm{H}-3,39-41$ & 11.19 & 3 & 66 & 18 & 32 \\
\hline 1H-2, 69-71 & 2.19 & 407 & 190 & 47 & 51 & $2 \mathrm{H}-3,49-51$ & 11.29 & 137 & 133 & 42 & 47 \\
\hline IH-2, 79-81 & 2.29 & 322 & 181 & 70 & 51 & $2 \mathrm{H}-3,59-61$ & 11.39 & 164 & 144 & 46 & 32 \\
\hline IH-2, 89-91 & 2.39 & 340 & 334 & 90 & 100 & $2 \mathrm{H}-3,69-71$ & 11.49 & 153 & 145 & 60 & 47 \\
\hline $1 \mathrm{H}-2,99-101$ & 2.49 & 204 & 254 & 49 & 55 & $2 \mathrm{H}-3,79-81$ & 11.59 & 123 & 125 & 73 & 41 \\
\hline $1 \mathrm{H}-2,109-111$ & 2.59 & 217 & 256 & 82 & 60 & $2 \mathrm{H}-3,89-91$ & 11.69 & 64 & 145 & 70 & 58 \\
\hline $1 \mathrm{H}-2,119-121$ & 2.69 & 124 & 180 & 50 & 49 & $2 \mathrm{H}-3,99-101$ & 11.79 & 136 & 181 & 59 & 48 \\
\hline $1 \mathrm{H}-2,129-131$ & 2.79 & 41 & 147 & 23 & 41 & $2 \mathrm{H}-3,109-111$ & 11.89 & 236 & 220 & 53 & 41 \\
\hline IH-2, 148-150 & 2.98 & 102 & $190^{\circ}$ & 69 & 71 & $2 \mathrm{H}-3,119-121$ & 11.99 & 157 & 209 & 53 & 43 \\
\hline $1 \mathrm{H}-3,9-11$ & 3.09 & 562 & 219 & 32 & 22 & $2 \mathrm{H}-3,129-131$ & 12.09 & 59 & 127 & 41 & 36 \\
\hline $1 \mathrm{H}-3,19-21$ & 3.19 & 419 & 147 & 49 & 34 & $2 \mathrm{H}-3,139-14 \mathrm{I}$ & 12.19 & 70 & 136 & 51 & 43 \\
\hline $1 \mathrm{H}-3,19-21$ & 3.19 & 432 & 140 & 43 & 37 & $2 \mathrm{H}-3,148-150$ & 12.28 & 27 & 63 & 27 & 31 \\
\hline $\mid \mathrm{H}-3,29-31$ & 3.29 & 183 & 124 & 44 & 38 & $2 \mathrm{H}-4,9-11$ & 12.39 & 15 & 57 & 17 & 38 \\
\hline $1 \mathrm{H}-3,39-41$ & 3.39 & 242 & 199 & 49 & 36 & $2 \mathrm{H}-4,19-21$ & 12.49 & 72 & 113 & 27 & 56 \\
\hline IH-3, 49-51 & 3.49 & 553 & 220 & 38 & 18 & $2 \mathrm{H}-4,29-31$ & 12.59 & 13 & 78 & 14 & 35 \\
\hline $1 \mathrm{H}-3,69-71$ & 3.69 & 241 & 173 & 67 & 54 & $2 \mathrm{H}-4,39-41$ & 12.69 & 206 & 124 & 40 & 46 \\
\hline IH-3, 79-81 & 3.79 & 348 & 172 & 84 & 34 & $2 \mathrm{H}-4,39-41$ & 12.69 & 220 & 125 & 42 & 44 \\
\hline IH-3, 89-91 & 3.89 & 296 & 156 & 59 & 60 & $2 \mathrm{H}-4,49-51$ & 12.79 & 325 & 167 & 72 & 34 \\
\hline $1 \mathrm{H}-3,99-101$ & 3.99 & 48 & 66 & 33 & 31 & $2 \mathrm{H}-4,59-61$ & 12.89 & 129 & 121 & 61 & 29 \\
\hline $1 \mathrm{H}-3,99-101$ & 3.99 & 47 & 64 & 32 & 34 & $2 \mathrm{H}-4,69-71$ & 12.99 & 68 & 110 & 97 & 43 \\
\hline $1 \mathrm{H}-3,109-11 \mathrm{I}$ & 4.09 & 85 & 59 & 26 & 18 & $2 \mathrm{H}-4,79-81$ & 13.09 & 96 & 86 & 122 & 61 \\
\hline$|\mathrm{H}-3| 19-121$, & 4.19 & 134 & 168 & 31 & 26 & $2 \mathrm{H}-4,89-91$ & 13.19 & 90 & 77 & 43 & 31 \\
\hline $1 \mathrm{H}-3,129-131$ & 4.29 & 61 & 128 & 31 & 48 & $2 \mathrm{H}-4,99-101$ & 13.29 & 22 & 127 & 24 & 34 \\
\hline $1 \mathrm{H}-3,139-141$ & 4.39 & 24 & 97 & 26 & 28 & $2 \mathrm{H}-4,109-111$ & 13.39 & 68 & 217 & 22 & 31 \\
\hline $1 \mathrm{H}-3,148-150$ & 4.48 & 24 & 109 & 14 & 37 & $2 \mathrm{H}-4,119-121$ & 13.49 & 87 & 226 & 22 & 29 \\
\hline $1 \mathrm{H}-4,19-2 \mathrm{I}$ & 4.69 & 34 & 83 & 23 & 19 & $2 \mathrm{H}-4,129-131$ & 13.59 & 57 & 172 & 17 & 41 \\
\hline $1 \mathrm{H}-4,29-31$ & 4.79 & 8 & 60 & 9 & 16 & $2 \mathrm{H}-4,139-141$ & 13.69 & 8 & 68 & 16 & 18 \\
\hline $1 \mathrm{H}-4,49-51$ & 4.99 & 233 & 128 & 32 & 13 & $2 \mathrm{H}-5,9-11$ & 13.89 & 6 & 45 & 15 & 11 \\
\hline $1 \mathrm{H}-4,59-61$ & 5.09 & 379 & 172 & 43 & 30 & $2 \mathrm{H}-5,19-2 \mathrm{I}$ & 13.99 & 31 & 122 & 32 & 21 \\
\hline $1 \mathrm{H}-4,69-71$ & 5.19 & 291 & 179 & 72 & 57 & $2 \mathrm{H}-5.29-3 \mathrm{I}$ & 14.09 & 121 & 148 & 46 & 27 \\
\hline $1 \mathrm{H}-4,89-91$ & 5.39 & 157 & 152 & 45 & 98 & $2 \mathrm{H}-5,39-4 \mathrm{I}$ & 14.19 & 221 & 96 & 74 & 48 \\
\hline 1H-4,99-101 & 5.49 & 18 & 76 & 15 & 55 & $2 \mathrm{H}-5,49-51$ & 14.29 & 139 & 89 & 96 & 45 \\
\hline $1 \mathrm{H}-4,109-111$ & 5.59 & 9 & 80 & 22 & 66 & $2 \mathrm{H}-5,59-61$ & 14.39 & 138 & 123 & 127 & 80 \\
\hline $1 \mathrm{H}-5,9-11$ & 6.09 & 5 & 130 & 22 & 25 & $2 \mathrm{H}-5,69-7 \mathrm{I}$ & 14.49 & 128 & 148 & 149 & 60 \\
\hline $1 \mathrm{H}-5,19-21$ & 6.19 & 38 & 137 & 31 & 24 & $2 \mathrm{H}-5,79-8 \mathrm{I}$ & 14.59 & 76 & 105 & 86 & 35 \\
\hline 1H-5, 29-31 & 6.29 & 323 & 158 & 29 & 28 & $2 \mathrm{H}-5,89-91$ & 14.69 & 75 & 111 & 51 & 32 \\
\hline 1H-5, 39-4l & 6.39 & 286 & 243 & 56 & 53 & $2 \mathrm{H}-5,99-101$ & 14.79 & 160 & 235 & 37 & 50 \\
\hline IH-5, 49-51 & 6.49 & 196 & 248 & 52 & 78 & $2 \mathrm{H}-5,109-111$ & 14.89 & 109 & 229 & 63 & 36 \\
\hline IH-5, 49-51 & 6.49 & 220 & 252 & 53 & 85 & $2 \mathrm{H}-5,119-121$ & 14.99 & 72 & 106 & 37 & 20 \\
\hline :H-5, 59-61 & 6.59 & 88 & 192 & 35 & 95 & $2 \mathrm{H}-5,129-131$ & 15.09 & 47 & 108 & 84 & 18 \\
\hline 1H-5, 69-71 & 6.69 & 85 & 171 & 59 & 103 & $2 \mathrm{H}-5,139-14 \mathrm{I}$ & 15.19 & 34 & 137 & 89 & 32 \\
\hline IH-5, 79-81 & 6.79 & 78 & 322 & 54 & 86 & $2 \mathrm{H}-6,9-11$ & 15.39 & 0 & 9 & 4 & 17 \\
\hline 1H-5, 89-91 & 6.89 & 64 & 201 & 54 & 91 & $2 \mathrm{H}-6,19-21$ & 15.49 & 18 & 49 & 11 & 45 \\
\hline $1 \mathrm{H}-5,99-101$ & 6.99 & 66 & 192 & 26 & 64 & $2 \mathrm{H}-6,32-34$ & 15.62 & 3 & 34 & 5 & 19 \\
\hline IH-5, 109-11। & 7.09 & 64 & 236 & 39 & 85 & $2 \mathrm{H}-6,39-41$ & 15.69 & 4 & 65 & 15 & 34 \\
\hline $1 \mathrm{H}-5,119-12 \mathrm{]}$ & 7.19 & 121 & 216 & 30 & 58 & $2 \mathrm{H}-6,49-5 \mathrm{I}$ & 15.79 & 24 & 130 & 17 & 18 \\
\hline IH-5, 119-121 & 7.19 & 128 & 218 & 31 & 57 & $2 \mathrm{H}-6,59-61$ & 15.89 & 59 & 70 & 17 & 13 \\
\hline IH-5, 129-131 & 7.29 & 0 & 23 & 4 & 10 & $2 \mathrm{H}-6,69-7 \mathrm{I}$ & 15.99 & 131 & 54 & 15 & 17 \\
\hline IH-5, 142-145 & 7.42 & 0 & 13 & 1 & 4 & $2 \mathrm{H}-6,79-81$ & 16.09 & 72 & 62 & 17 & 22 \\
\hline 1H-5, 149-151 & 7.49 & 1 & 23 & 1 & 9 & $2 \mathrm{H}-6.89-91$ & 16.19 & 0 & 12 & 10 & 11 \\
\hline $2 \mathrm{H}-1,9-11$ & 7.89 & 2 & 9 & 3 & 6 & $2 \mathrm{H}-6,99-101$ & 16.29 & 8 & 29 & 14 & 28 \\
\hline $2 \mathrm{H}-1,19-21$ & 7.99 & 65 & 197 & 61 & 110 & $2 \mathrm{H}-6,109-111$ & 16.39 & 1 & 28 & 9 & 32 \\
\hline $2 \mathrm{H}-1,29-3 \mathrm{H}$ & 8.09 & 37 & 153 & 43 & 63 & $2 \mathrm{H}-6,119-121$ & 16.49 & 2 & 78 & 9 & 27 \\
\hline $2 \mathrm{H}-1,39-41$ & 8.19 & 0 & 10 & 5 & 7 & $2 \mathrm{H}-6,129-131$ & 16.59 & 20 & 128 & 9 & 26 \\
\hline $2 \mathrm{H}-1,49-51$ & 8.29 & 6 & 104 & 53 & 30 & $2 \mathrm{H}-6,139-141$ & 16.69 & 2 & 59 & 12 & 20 \\
\hline $2 \mathrm{H}-1,49-51$ & 8.29 & 4 & 101 & 36 & 43 & $2 \mathrm{H}-6,148-150$ & 16.78 & 0 & 46 & 12 & 28 \\
\hline $2 \mathrm{H}-1,59-61$ & 8.39 & 48 & 204 & 56 & 40 & $2 \mathrm{H}-7,9-11$ & 16.89 & 0 & 27 & 7 & 28 \\
\hline $2 \mathrm{H}-1,69-71$ & 8.49 & 53 & 189 & 56 & 26 & $2 \mathrm{H}-7,19-2 \mathrm{I}$ & 16.99 & 7 & 62 & II & 30 \\
\hline $2 \mathrm{H}-1,79-8 \mathrm{I}$ & 8.59 & 116 & 194 & 83 & 40 & $2 \mathrm{H}-7,32-34$ & 17.12 & 58 & 241 & 13 & 46 \\
\hline $2 \mathrm{H}-1,89-91$ & 8.69 & 18 & 88 & 63 & 35 & $2 \mathrm{H}-7,39-41$ & 17.19 & 66 & 232 & 17 & 67 \\
\hline $2 \mathrm{H}-1,99-101$ & 8.79 & 39 & 104 & 74 & 43 & $2 \mathrm{H}-7,39-41$ & 17.19 & 62 & 232 & 18 & 62 \\
\hline $2 \mathrm{H}-1,109-111$ & 8.89 & 27 & 90 & 63 & 45 & $2 \mathrm{H}-7,49-51$ & 17.29 & 23 & 92 & 27 & 49 \\
\hline $2 \mathrm{H}-1,119-121$ & 8.99 & 33 & 157 & 65 & 65 & $2 \mathrm{H}-7,59-61$ & 17.39 & 8 & 41 & 25 & 58 \\
\hline $2 \mathrm{H}-1,129-131$ & 9.09 & 33 & 178 & 64 & 62 & $2 \mathrm{H}-7,69-71$ & 17.49 & 13 & 53 & 19 & 56 \\
\hline
\end{tabular}

*ODP depth assignment $=$ depth has not been adjusted. 